\title{
Mitochondrial protease Lonp1 localizes to the nucleus in response to heat shock
}

\section{Lara Gibellini}

University of Modena and Reggio Emilia: Universita degli Studi di Modena e Reggio Emilia

\section{Rebecca Borella}

University of Modena and Reggio Emilia: Universita degli Studi di Modena e Reggio Emilia

\section{Anna De Gaetano}

University of Modena and Reggio Emilia: Universita degli Studi di Modena e Reggio Emilia

\section{Giada Zanini}

University of Modena and Reggio Emilia: Universita degli Studi di Modena e Reggio Emilia

\section{Domenico Lo Tartaro}

University of Modena and Reggio Emilia: Universita degli Studi di Modena e Reggio Emilia

\section{Gianluca Carnevale}

University of Modena and Reggio Emilia: Universita degli Studi di Modena e Reggio Emilia

\section{Francesca Beretti}

University of Modena and Reggio Emilia: Universita degli Studi di Modena e Reggio Emilia

\section{Lorena Losi}

University of Modena and Reggio Emilia: Universita degli Studi di Modena e Reggio Emilia

\section{Sara De Biasi}

University of Modena and Reggio Emilia: Universita degli Studi di Modena e Reggio Emilia

\section{Milena Nasi}

University of Modena and Reggio Emilia: Universita degli Studi di Modena e Reggio Emilia

\section{Mattia Forcato}

University of Modena and Reggio Emilia: Universita degli Studi di Modena e Reggio Emilia

\section{Andrea Cossarizza}

University of Modena and Reggio Emilia: Universita degli Studi di Modena e Reggio Emilia

Marcello Pinti ( $\sim$ marcello.pinti@unimore.it )

Universita degli Studi di Modena e Reggio Emilia https://orcid.org/0000-0001-9118-1262

\section{Research Article}

Keywords: Mitochondria, Lonp1, HSF1, heat shock

Posted Date: February 24th, 2021 
DOl: https://doi.org/10.21203/rs.3.rs-227270/v1

License: (c) (1) This work is licensed under a Creative Commons Attribution 4.0 International License. Read Full License 
Mitochondrial protease Lonp1 localizes to the nucleus in response to heat shock

Lara Gibellini ${ }^{1}$, Rebecca Borella ${ }^{1}$, Anna De Gaetano ${ }^{2}$, Giada Zanini ${ }^{2}$, Domenico Lo Tartaro ${ }^{1}$, Gianluca Carnevale ${ }^{3}$, Francesca Beretti ${ }^{3}$, Lorena Losi ${ }^{2}$, Sara De Biasi ${ }^{1}$, Milena Nasi ${ }^{3}$, Mattia Forcato $^{2}$, Andrea Cossarizza ${ }^{1}$, Marcello Pinti2 ${ }^{\star}$

${ }^{1}$ Dept. of Medical and Surgical Sciences for children and Adults, University of Modena and Reggio Emilia, Modena, Italy

${ }^{2}$ Dept. of Life Sciences, University of Modena and Reggio Emilia, Modena, Italy

${ }^{3}$ Dept. of Surgical, Medical, Dental and Morphological Sciences, University of Modena and Reggio Emilia, Modena, Italy

${ }^{*}$ Corresponding author:

Marcello Pinti, Ph.D.

Department of Life sciences,

University of Modena and Reggio Emilia

Via Campi 287

41125 Modena

T. +390592055386

F. +390592055426

E-mail: marcello.pinti@unimore.it 


\section{Abstract}

The coordinated communication between the mitochondria and nucleus is essential for cellular activities. Nonetheless, the pathways involved in this crosstalk are scarcely understood. The protease Lonp1 was previously believed to be exclusively located in the mitochondria, with an important role in mitochondrial morphology, mtDNA maintenance, and cellular metabolism, in both normal and neoplastic cells. However, we recently detected Lonp1 in the nuclear, where as much as $22 \%$ of all cellular Lonp1 can be found. Nuclear localization is detectable under all conditions, but the amount is dependent on a response to heat shock (HS). Lonp1 in the nucleus interacts with heat shock factor 1 (HSF1) and modulates the HS response. Furthermore, we found three splicing variants of Lonp1 mRNA that coexist in cells and generate three Lonp1 isoforms (ISO1, ISO2 and ISO3) with differential subcellular distributions. All three isoforms move into the nucleus under $\mathrm{HS}$, and ISO2 is most frequently upregulated in neoplastic cells compared to ISO1 and ISO3. These findings reveal a novel extramitochondrial function for Lonp1 in response to stress.

\section{Keywords}

Mitochondria, Lonp1, HSF1, heat shock 


\section{Abbreviations}

ATFS-1, activating transcription factor associated with stress 1

CODAS, Cerebral Ocular Dental Auricular Skeletal Anomalies Syndrome

DDR, DNA damage response

DMEM, Dulbecco modified Eagle medium

DOXO, doxorubicin

eGFP, enhanced green fluorescent protein

FBS, foetal bovine serum

HS, heat shock

HSF1, heat shock factor 1

HSP70, Heat Shock Protein-70

hspQ, HS protein $Q$

HSR, HS response

HU, hydroxyurea

$\mathrm{IR}$, ionizing radiation

IP, immunoprecipitation

ISO, isoform

LMB, leptomycin B

MAMs, mitochondrial associated membranes

mtDNA, mitochondrial DNA

MSigDB, reactome collection of Molecular Signature Database

MTS, Mitochondrial Targeting Sequence

MW, molecular weight

NLS, nuclear localization signal

NRF2, nuclear factor erythroid 2-related factor 2

PBS, phosphate buffered saline 
PCA, principal component analysis

PDC, pyruvate dehydrogenase complex

PFA, paraformaldehyde

RIPA, Radioimmunoprecipitation assay buffer

ROS, reactive oxygen species

SDS, sodium dodecyl sulfate

siRNA, small interfering RNA

TA, transient amplifying

UPR, unfolded protein response 


\section{Introduction}

Recently, there has been increasing evidence that mitochondria not only communicates with the nucleus but that several proteins containing mitochondrial-targeting sequences also reside or translocate to the nucleus under certain conditions (1). These proteins can translocate in response to several stimuli and likely perform a similar function in both mitochondrial and nuclear compartments (1). Examples of mitochondrial protein moonlighting to the nucleus include: mammalian nuclear factor erythroid 2-related factor 2 (NRF2), Activating Transcription Factor associated with Stress 1 (ATFS-1), the monooxygenase CLK-1, the pyruvate dehydrogenase complex (PDC), and fumarase (1-4).

Lonp1 is a mitochondrial protease which plays a crucial role in the maintenance of mitochondrial functions (5). Three Lonp1 variants are present in humans, resulting from the alternative splicing of exon 1. Global loss of Lonp1 causes fragmentation of the mitochondrial network, associated with severe alterations of cristae, as well as mitochondrial membrane depolarization and reduced respiratory capacity $(6,7)$. On the contrary, Lonp1 overexpression is associated with unchanged mitochondrial morphology and mass (8). Changes in Lonp1 expression have been linked to epithelial-mesenchymal transition in cancer (8-10), and mutations in the Lonp1 sequence have been linked to cerebral, ocular, dental, auricular and skeletal syndromes $(5,11)$. In mitochondria, Lonp1 is mainly involved both in the degradation of misfolded, oxidatively modified and regulatory proteins, and in the preservation of mitochondrial DNA (mtDNA) (12-14). Recently however, Lonp1 has also been reported to play a role in metabolism remodelling (7). This indicates that additional extra-mitochondrial functions of Lonp1 may exist (15). As previous assumptions have believed that Lonp1 is located exclusively in the mitochondria, possible nuclear roles of this protein have been paid little attention. Here we provide evidence that Lonp1 can be present in the nuclei of murine and human tissue and cultured human cells. The nuclear localization 
of Lonp1 is increased in response to heat shock (HS) is independent of either DNA damage or cell cycle. Furthermore, we report that three isoforms of Lonp1 coexist in the cells, and that all the isoforms can localize in the nucleus in response to HS.

\section{Materials and Methods}

\section{Mice}

Lonp $1^{\text {wt- }}$ mice were generated with a knock-out allele encompassing exons 5 and 8 from Biogem Srl (Ariano Irpino, Italy) on a C57BL/6 background (16). The genotyping was performed on genomic DNA extracted from ear samples and obtained using the commercial Easy-DNA $^{\text {TM }}$ gDNA Purification Kit (Thermo Fisher Scientific, Waltham, MA, USA). 20-50 ng of DNA were amplified in $25 \mu \mathrm{L}$ of a reaction mix containing $1 \mathrm{X}$ GoTaq Flexy Buffer (Promega Corporation, Madison, WI, USA), 2 mM MgCl $2,0.2 \mathrm{mM}$ of each dNTP (Promega), $0.03 \cup$ of GoTaq G2 Flexy DNA Polymerase and $0.2 \mu \mathrm{M}$ of the following primers: forward 5CAGGGAAGAAACTGAAGTCAGGC-3, reverse 5-CACTCTGGTTCATGGCCACC-3. A 925 bp PCR product was obtained only for knock-out allele. All mice were housed in ventilated cages for a 12-hrs day/night cycle with access to water and food ad libitum. Animal protocols were approved by the Italian law protecting animals used for scientific purposes (authorization $n^{\circ} 253 / 2017-P R$, released on March, $21^{\text {st }}, 2017$ from Italian Ministry of Health).

\section{Cell Culture and transfections}

SW620 were maintained in Glutamax RPMI 1640 supplemented with gentamycin and 10\% foetal bovine serum (FBS). SW480 and HeLa cells were maintained in Glutamax Dulbecco modified Eagle medium (DMEM) supplemented with gentamycin and $10 \%$ FBS (Life 
Technologies). Cells were maintained in an incubator at $37^{\circ} \mathrm{C}, 5 \% \mathrm{CO} 2$, in a humidified atmosphere. To induce heat shock, cells were incubated in a water bath set at $42^{\circ} \mathrm{C}$. Plasmid DNA transfections were performed with Lipofectamine 3000 (Life Technologies).

\section{Immunofluorescence and confocal microscopy}

Cells for confocal microscopy were grown on coverslips, fixed with $4 \%$ paraformaldehyde (PFA, Sigma Aldrich) in PBS, for 10 minutes, and permeabilized with $0.1 \%$ triton $\mathrm{X}-100$ in PBS, for 15 minutes. Cells were blocked in $3 \%$ bovine serum albumin (BSA) for 1 hour, incubated with primary antibodies overnight, washed, incubated with Alexa Fluor-conjugated secondary antibodies (Life Technologies) in 3\% BSA for 1 hour, and washed again to remove unbound antibody. Cells were counterstained with DAPI. Coverslips were mounted in Fluoromount (Sigma Aldrich) and images were collected on a confocal microscope SP8AOBS (Leica). Image analysis was performed with ImageJ and ScanR.

\section{Western blotting}

Western blotting was performed as previously described (6). Total cell lysates were prepared in RIPA lysis buffer plus protease inhibitors cocktail (Sigma Aldrich) and phosphatase inhibitors (Sigma Aldrich). Nuclear fractions were obtained by using NE-PER nuclear and cytoplasmic extraction kit and following manufacturer's instructions. Samples were resolved by SDS-PAGE on precast gels $(12 \%, 4-12 \%)$ and transferred to nitrocellulose membranes (Bio-Rad Laboratories), which were then immunoblotted. The following primary antibodies were used: custom anti-Lonp1 (Primm), anti-Lamin B1 (Santa Cruz Biotechnology), anti- $\beta$-actin (Abcam), anti-TOM20 (Santa Cruz Biotechnology), anti-SIRT3 (Santa Cruz Biotechnology), anti-GFP (Abcam), anti-HSF1 (Abcam). The following secondary antibodies were used: HRP-conjugated goat anti-rabbit and HRP-conjugated goat anti-mouse (Bio-Rad Laboratories). Enhanced Clarity chemiluminescent substrate 
(Bio-Rad laboratories) was used to detect proteins by using a Chemidoc MP (Bio-Rad Laboratories). Image analysis was performed by Image Lab software v5.2.1.

\section{Immunoprecipitation}

For immunoprecipitation (IP) experiments we used GFP Trap agarose beads (Chromotek, Germany) according to the manufacturer's protocol. 800 ugr of total lysate was incubated with GFP Trap Agarose beads (Chromotek, Germany) overnight at $4^{\circ} \mathrm{C}$ with constant rotation in a RIPA modified Buffer (20umM Tris-Cl, pH 7.0; 1\% Nonidet P-40; 150amM NaCl; 10\% glycerol; 10umM EDTA; 20umM NaF; 5umM sodium pyrophosphate; and 1amM Na3VO4). Beads were washed three times with wash buffer (20umM Tris-Cl, $\mathrm{pH} 7.0,1 \%$ Nonidet P-40, 150umM NaCl, 10\% glycerol, 10umM EDTA, 20umM NaF, 5umM sodium pyrophosphate), and once with $10 \mathrm{amM}$ Tris- $\mathrm{Cl}, \mathrm{pH}$ 7.4. The target proteins bound were eluted from beads by boiling in SDS loading buffer for $5 \mathrm{~min}$ at $99^{\circ} \mathrm{C}(17)$.

\section{Histology and immunohistochemistry}

Histological sections of mice colon $(n=3)$ were deparaffinized in xylene and rehydrated in alcohol upon distilled water. Antigen retrieval was performed by incubating slides in $10 \mathrm{mM}$ sodium citrate buffer, $\mathrm{pH} 6.0$, at sub-boiling temperature for 15 minutes. Then, endogenous peroxidase activity was blocked by incubating sections in a $5 \%$ methanol plus $1 \%$ hydrogen peroxide solution for 5 minutes. After blocking with PBS containing 3\% BSA, sections were incubated overnight at $4^{\circ} \mathrm{C}$ with the primary rabbit anti-Lonp1 antibody diluted 1:100 in PBS containing 3\% BSA. Then, after 3 washings, HRP conjugated secondary antibody was incubated (diluted 1:200 in PBS) for 1 hour at room temperature. Finally, after 3 washings in PBS, HRP was revealed by a DAB based kit (Sigma Aldrich), according to manufacturers' instructions. 


\section{Flow cytometry}

Cell cycle was analysed by flow cytometry. In brief, cells were treated with $10 \mu \mathrm{M}$ 5-ethynyl2'-deoxyuridine (Edu) for $2 \mathrm{hrs}$. Cells were detached and immediately stained using to ClickiT $^{\mathrm{TM}}$ EdU Alexa Fluor ${ }^{\mathrm{TM}} 488$ Flow Cytometry Assay Kit (Thermo Fisher Scientific), following manufacturer protocol/standard procedures. Fixed cells were acquired by Attune NxT acoustic flow cytometer (Thermo Fisher Scientific) equipped with four lasers (405 nm, 488 $\mathrm{nm}, 561 \mathrm{~nm}, 638 \mathrm{~nm}$ ). A minimum of 10,000 events were acquired for every sample (18).

\section{RNAseq}

SW620 cells exposed to heat shock for and RNA extracted using RNeasy mini kit (QIAgen) following provided instructions. Three independent biological replicates were examined. The quality of RNA samples was checked by using an Agilent 2100 Bioanalyzer (Agilent, Santa Clara, CA, USA); RNA samples were used either for RNA-seq and for qPCR validation. RNAseq library was prepared from $1.5 \mu \mathrm{g}$ of total RNA using TruSeq Stranded mRNA Library Prep Kit in paired end format (Illumina) following the manufacturer's recommendations. RNA sequencing was carried out on Illumina Novaseq 6000 (Illumina) at BMR Genomics (Padua, Italy).

\section{Bioinformatics analysis}

Raw reads were aligned using STAR version 2.7.3a to build version hg38 of the human genome (19). Counts for UCSC annotated genes were calculated from the aligned reads using featureCounts function of the Rsubread R package (20) and R (version 3.6.3). Normalization and differential analysis were carried out using edgeR $R$ package (21). Raw counts were normalized to obtain Counts Per Million mapped reads (CPM). Principal component analysis (PCA) was performed using the top $10 \%$ variable genes and the prcomp function of stats R package. Only genes with a CPM greater than 1 in at least 3 samples 
were retained for differential analysis. Gene expression changes were considered statistically significant with Benjamini-Hochberg FDR less than 5\% and fold changes greater than 2 for upregulation and lower than -2 for downregulation.

Functional enrichment analysis of the differentially expressed genes was performed using Enrichr (https://maayanlab.cloud/Enrichr/) and DAVID (Version 6.8, Oct 2016; https://david.ncifcrf.gov/home.jsp) (22-25). Gene set enrichment analysis version X (26)was performed on the log2 TMM values calculated by edgeR and the Reactome collection of Molecular Signature Database (MSigDB) version 7.2 (27). Gene sets were considered significantly enriched at FDR $\leq 5 \%$ when using 1,000 permutations of gene sets. RNA-seq data from this study have been deposited at Gene Expression Omnibus database (GEO, https://www.ncbi.nlm.nih.gov/geo/) with accession number GSE164834.

\section{Statistical analysis}

All the measurements data are presented as mean \pm standard deviation (SD) if not differently specified. Statistical analysis between different experimental conditions was performed with ANOVA followed by the Bonferroni means comparison or $t$ test when appropriate. Unpaired $t$ test was used to compare the levels of the different isoforms between normal and tumour tissues in different cancer types. A threshold of $P \leq 0.05$ was selected to indicate statistical significance. Statistical calculations were performed using standard functions of GraphPad Prism 8.0. Image analysis was performed by ImageJ v2.0 and ScanR. 


\section{Results}

\section{Lonp1 localization in mitochondria and nuclei}

Previous data from our lab revealed that mitochondrial Lonp1 is overexpressed in colon cancer cells and tissue, and that it is involved in energetic metabolism and epithelialmesenchymal transition in these cells $(6-8,28)$. Lonp1 was previously assumed to localize exclusively in mitochondria. Intriguingly, while analysing the tissue-specific expression of Lonp1 in mice, we identified the nuclear localisation of Lonp1 in murine gut epithelium (Figure 1), confirmed by immunofluorescence and immunohistochemistry. Surprisingly, we observed Lonp1 expression in the nuclei of healthy gut cell samples from adult wild-type mice (Figure 1A), confirmed by immunofluorescence (Figure 1B). Ki-67 staining enabled the observation of section crypts into the lower $(0$ to +4$)$, upper $(+5$ to +15$)$ and non- $(>+15$; Figure 1C) crypt zones. Detailed analysis of Lonp1 nuclear expression within the crypts showed that positivity of Lonp1 was concentrated in the transient amplifying (TA) cell area $(+5$ to +15 ; Figure $1 \mathrm{C})$. We next searched for nuclear localization signal (NLS) in a murine Lonp1 aminoacidic sequence with cNLS Mapper (available at http://nlsmapper.iab.keio.ac.jp/cgi-bin/NLS_Mapper_form.cgi). A putative NLS sequence (RRKLKRGKKEVE) was identified in Lonp1 between amino acid 225 and 236. This sequence is conserved in human Lonp1 (RRKSKRGKKEAE), as well as in other mammals (Figure 1D). We therefore analysed Lonp1 subcellular localization in human colon tissue, and also found Lonp1 human nuclear localisation (Figure 1E; indicated by arrows).

To verify the nuclear presence of Lonp1 in neoplastic cells, we next examined Lonp1 subcellular localization in human cell lines. As shown in Figure $2 \mathrm{~A}$, we found that endogenously expressed Lonp1 is present both in mitochondria and nuclei of SW620 cells. The quantification of Lonp1 expression in nuclear and cytosolic fractions obtained from 
SW620 cells also revealed that 10 to $22 \%$ of Lonp1 is present in the nucleus of these cells (Figure 2B and 2C). Lonp1 nuclear localization was also confirmed in two other colon cancer cell lines, namely SW48 and SW480, and in HeLa cells (Supplementary Figure 1). Subsequently, we treated cells overnight with $50 \mathrm{nM}$ leptomycin B (LMB), which inhibits nuclear export. The expression and subcellular distribution of p62 was used as a positive control to confirm the effectiveness of LMB treatment. In this condition, decreased levels of Lonp1 were observed in the cytoplasm, whereas increased levels were observed in the nucleus of treated cells (Figure 2D and 2E), indicating that a fraction of cytosolic Lonp1 translocates into the nucleus.

\section{Lonp1 localizes to the nucleus in response to heat shock}

The dual localization of Lonp1 in the mitochondria and the nucleus may be part of a regulatory mechanism that links mitochondrial function to nuclear events. To identify the potential functions of the nuclear protein and considering that Lonp1 nuclear localization correlates with Ki-67 expression, at least in mice, we first investigated whether Lonp1 could be involved in cell cycle regulation. For this reason, SW620 cells were exposed to thymidine, which causes cell cycle arrest in G0/G1, and Lonp1 cell localization was assessed (Figure 3A). No changes were observed in the nuclear fraction of Lonp1 in thymidine treated cells compared to untreated cells. This observation ruled out the involvement of Lonp1 in cell cycle regulation.

Since Lonp1 is able to bind to DNA within the mitochondria, we next investigated whether Lonp1 was recruited to the nucleus due to DNA damage response (DDR) (Monaghan and Whitmarsh, 2015). To examine the effects of double-strand breaks on Lonp1 expression, SW620 cells were exposed to 5 Gy of ionizing radiation (IR) and left to recover for 24 hours. We first confirmed that DNA damage occurred in our model, by analysing the levels of $\gamma-\mathrm{H} 2 \mathrm{AX}$, a key player in of the DDR in irradiated cells. As shown in 
Figure 3B, the $\gamma-\mathrm{H} 2 \mathrm{AX}$ protein level was significantly increased in irradiated SW620 cells compared to untreated cells, and the presence of the $\gamma-\mathrm{H} 2 \mathrm{AX}$ foci was also confirmed (left panel). However, irradiated cells did not show any changes in Lonp1 subcellular distribution when compared to untreated cells, suggesting that nuclear Lonp1 is not related to a DNA damage response (Figure 3B, right panel). These results were confirmed using other DNA damage inducing mechanisms of hydroxyurea $(\mathrm{HU})$ or doxorubicin (DOXO) treatment. Cells were treated with increasing concentrations of these molecules for varying exposure times, and the levels of $\gamma-\mathrm{H} 2 \mathrm{AX}$ were analysed. We found that treatment with $4 \mathrm{mM} \mathrm{HU}$ or $2 \mu \mathrm{M}$ DOXO led to $\gamma-\mathrm{H} 2 \mathrm{AX}$ increase, and to the appearance of $\gamma-\mathrm{H} 2 \mathrm{AX}$ foci in the nucleus (Figure 3C). Therefore, under these conditions the levels of Lonp1 in nuclear and cytosolic fractions from cells were measured, but no changes in Lonp1 subcellular distribution were observed (Figure 3C, right panel). Despite their diverse mechanisms of action, these stressors had no effect on Lonp1 subcellular localization, clearly indicating that DNA damage does not induce Lonp1 localization.

An alternative hypothesis was that Lonp1 translocates to the nucleus in response to proteotoxic stress. As heat shock is commonly used to induce proteotoxic stress (29), we kept SW620 cells for up to 3 hours at $42^{\circ} \mathrm{C}$ and then left them to recover. We then analysed Lonp1 expression and distribution and observed that there was a clear localization of Lonp1 in the nucleus of treated cells compared to an insignificant presence in the nucleus of control cells (Figure 4A). We then analysed the expression of Lonp1 in cell nuclear and cytosolic fraction under HS compared to controls. A successful induction of HS was confirmed through the observation of an increase in the expression of Heat Shock Protein-70 (HSP70), both in the nucleus and in the cytoplasm. Lonp1 expression in the same fraction showed a 4-fold increase of the protein in the nucleus of treated cells when compared to controls, indicating that HS induces Lonp1 relocalisation to the nucleus (Figure 4B and 4C). 
We hypothesized that Lonp1 in the nucleus may contribute to regulate HS response (HSR). In mammalian cells, HSR is regulated by heat shock factor-1 (HSF1) (Wu, 1995). While HSF1 is mostly located in the cytosol and nucleus of unstressed cells, it accumulates in the nucleus in heat-shocked cells and promotes the transcription of HSR genes. Thus, we assumed that Lonp1 may interact with HSF-1 and modulate its function in the nucleus. We first looked for an interaction between Lonp1 and HSF1. Therefore, SW620 cells were transfected with a full-length form of Lonp1 tagged with eGFP at C-term, heat-shocked for 3 hours, and left to recover. Protein extracts were pulled down with anti-GFP Ab and assayed with anti HSF1 Ab (Figure 4D), showing that under normal conditions physically interacts with Lonp1, and that HS increased the affinity of Lonp1 for HSF1. The interaction is not only present for the eGFP tagged Lonp1 (Figure 4D), but also for the endogenous protein, excluding a possible artefact. The same extracts pulled down with anti HSF-1 and tested with anti GFP Ab confirmed the specificity of the interaction (Figure 4E). Knock-down of Lonp1 followed by HS and recovery determined an increase in the levels of HSF1 in the nucleus (Figure 4F), suggesting that Lonp1 can modulate HSF1 levels, most likely by degrading it. In addition, when Lonp1 was silenced by small interfering RNA (siRNA), the levels of HSP70 increased both during HS and recovery (Figure 4G).

In order to elucidate the relationship nuclear Lonp1 and HSF1, we performed an RNAseq analysis on SW620 cells with silenced Lonp1 and undergoing HS, or HS followed by recovery. As PCA and cluster analysis showed a close similarity between HS and HS plus recovery samples (Supplementary figure 2), we focused our attention only on HS.

In HS condition, 327 genes were upregulated and 148 downregulated in cells treated with siLonp 1 when compared to cells kept at $37^{\circ} \mathrm{C}$. In cells treated with a scramble siRNA, HS determined the upregulation of 376 genes and the downregulation of 47 genes (Figure 5A). Genes upregulated during HS in Lonp1-silenced cells are largely overlapping with those upregulated in cells treated with a scramble siRNA, but 125 of them (25\%) are 
uniquely upregulated when Lonp1 was silenced, indicating that silencing of Lonp1 modified HS response (Figure 5B).

As expected, gene ontology analysis revealed that, when compared to control samples, genes upregulated in HS were significantly enriched in "response to unfolded protein", "protein refolding" and "regulation of cellular response to heat" biological processes, either in cells treated with a Lonp1 siRNA or with a scrambled, control siRNA (Supplementary Table1).

A significant enrichment of genes related to "regulation of HSF1 mediated HS response" "HSF1 dependent transactivation" and "HSF1 activation" pathways was also observed (Supplementary table 2). Thus, we focused our attention on HSF1 target genes during HS, and we compared the response to HS when Lonp1 was silenced or not.

HSF1 target genes showed a slightly higher expression in samples under HS where Lonp1 was silenced, if compared to samples under HS treated with a scrambled siRNA, with the only notable exception of HSPA1A (Supplementary Table 3) (30). This suggests that the absence of Lonp1 determined a stronger response to HS, mediated by HSF1. GSEA analysis confirmed this observation (Figure 5 and supplementary table 4). Samples kept at $37^{\circ} \mathrm{C}$ did not show any relevant enrichment of HSF 1 target genes when Lonp1 is silenced if compared to samples treated with scrambled siRNA (Figure 5C, left panel). Conversely, HS determined a slight, but significant enrichment of HSF1 target genes in Lonp1-knocked down cells (Figure 5C, right panel). Overall, these data indicate that Lonp1 knock down leads to a further increase of HSF1 target genes at the transcriptional level in response to HS, and suggest that Lonp1 in the nucleus interacts with HSF1, modulating the HSR.

\section{Lonp1 isoforms show differential expression pattern and intracellular distribution}

The human Lonp1 isoform-1 protein (ISO1) is formed by 959 amino acids and has been highly conserved throughout evolution. However, at least two other isoforms are 
present in humans, resulting from alternative splicing of exon 1 (Figure 6A). Lonp1 Isoform2 (ISO2) is 895-aminoacid long and lacks aminoacids 42-105. Lonp1 isoform-3 (ISO3) contains 763 aminoacids and lacks aminoacids 1-196, including the Mitochondrial Targeting Sequence (MTS). NLS is present in all Lonp1 isoforms.

We therefore investigated if these three isoforms have a different expression level and/or intracellular distribution. We explored the in silico data present in the TSV database (TSVdb), a database reporting the relative levels of mRNA splicing variants; ISO2 expression is almost 3-fold higher than ISO1, regardless of the type of cells taken into account, while ISO3 was only detectable in bladder and head-and-neck cancer cells (Figure 6B).

Thus, we conjectured that these three isoforms display different intracellular distributions, and may localize in the nucleus independently. We therefore generated three constructs where each isoform was tagged at the C-term by eGFP or mCherry, and we analysed the intracellular distribution in transfected SW620 cells. When cell lysates were probed with an anti-eGFP Ab, ISO1-eGFP and ISO2-eGFP the same MW of approximately 138 KDA was observed, which was slightly higher than predicted (Figure 6C, left panel) (Wang et al., 1993). EGFP-ISO3 was lighter than ISO1-eGFP and ISO2-eGFP, with a MW of $112 \mathrm{KDa}$ (Figure 6C, left panel). The same samples were also probed with anti-Lonp1 $\mathrm{Ab}$ (Figure 6C, right panel). This Ab correctly detected the bands corresponding to the three Lonp1-eGFP isoforms, but only evidenced the band corresponding to the endogenous ISO1 and ISO2 Lonp1, suggesting that the levels of ISO3 is normally very low and could not be detected. Then, we again transfected cells with eGFP-tagged isoforms and assayed nuclear and mitochondrial fractions with anti-eGFP and anti Lonp1 antibodies. All the isoforms were present in the nuclear and cytosolic fractions (Figure 6D, left panel), while ISO3-eGFP was barely detected in the mitochondrial fraction (Figure 6D, right panel). 
Consistently, confocal microscopy analysis showed that ISO1 was mainly mitochondrial, ISO2 was mainly mitochondrial and partially nuclear, while ISO3 was cytosolic and nuclear but not mitochondrial, in agreement with the absence of the MTS (Figure 6E). When cells were co-transfected with ISO1-mCherry and ISO2-eGFP, a clear colocalization of both proteins in mitochondria was observed (Supplementary Figure 3). On the contrary, when cells were co-transfected with ISO1-mCherry and ISO3-eGFP, colocalization was absent (Supplementary Figure 3).

Then, we asked whether a difference may be present among different isoforms to translocate in the nucleus in response to HS. Thus, cells were transfected with the eGFPtagged isoforms of Lonp1 and analysed for the nuclear levels of the protein after 3 hours of HS, or HS followed by recovery (Figure 7A). As shown in Figure 7B, ISO1 nuclear levels showed a 2-fold increase, both after HS and HS plus recovery whereas no significant change was observed in the cytosolic fraction. Similarly, ISO2 and ISO3 forms showed a 2-fold nuclear increase, which were significant in the case of ISO3 in HS condition. Confocal microscopy analysis confirmed that all three forms can relocate to the nucleus under HS conditions (Figure 7C). Overall, these data strongly suggested that the nuclear redistribution of Lonp1 in response to HS was independent of the individual isoforms.

\section{Lonp1 isoforms show differential expression between cancer and normal tissue}

Alternative splicing is a tightly regulated process allowing for the expression of multiple RNA and protein isoforms from one gene, and its alteration has recently been described in several diseases, including cancer (31). Thus, we analysed data available in the TSVdb concerning the expression of Lonp1 isoforms in different primary solid tumours compared with normal tissue (Figure 7D). We found that ISO1 was upregulated in lung, bladder, prostate and breast cancer (left panel), and ISO2 was upregulated in all the other types of cancers we analyzed, i.e. rectum, colon, cervical, bladder, prostate, breast, head 
and neck and renal (right panel, and Supplementary Figure 4). Conversely, the expression of ISO3 did not show any significant changes in any types of cancer considered (data not shown).

\section{Discussion}

Lonp1 is a nuclear-encoded mitochondrial enzyme, previously assumed to modulate several cellular functions, exclusively in the mitochondria. Inactivation of Lonp1 by small interfering RNA (siRNA) or by specific inhibitors, has been proven to lead to defective respiration, alteration of mitochondrial ultrastructure and inhibition of mitochondrial translation $(6,32,33)$. For the first time, this study demonstrates that Lonp1 is also expressed in the nucleus, that its relocalisation is in response to $\mathrm{HS}$ and that all Lonp1 isoforms relocate in the nucleus in response to HS.

As no other mitochondrial protease has been observed in the nucleus, the localization of Lonp1 in this study was surprising but not totally unexpected, for several reasons. Firstly, an extra-mitochondrial form of Lonp1 has already been demonstrated by Polo et al., who identified mitochondrial associated membranes (MAMs) as a site where Lonp1 localizes in the presence of ER-stress, clearly indicating that Lonp1 is not exclusively mitochondrial (15). Independent observations in other cellular and animal models have also confirmed this observation $(34,35)$. Secondly, several mitochondrial proteins have been shown to relocate to the nucleus in response to different stimuli, and to regulate a plethora of functions. The monooxygenase CLK-1 is a mitochondrial enzyme involved in respiration and longevity. However, a nuclear, independent form of this protein exists and mediates a retrograde signal in response to mitochondrial Reactive Oxygen Species (ROS). Similarly, ATFS-1, a transcription factor that regulates mitochondria-to-nuclear communication during the $\mathrm{mt}$ Unfolded protein response (UPR), can move to mitochondria and, in condition of 
mitochondrial stress, to the nucleus, where it regulates the transcription of OXPHOS related genes. Although the behaviour of Lonp1 is very similar to that of CLK-1 and ATFS-1, a crucial difference exists; both the nuclear and mitochondrial form of Lonp1 is identical whereas the nuclear CLK-1 and ATFS-1 (include the MTS) and therefore differ from the mitochondrial forms of the respective proteins. Therefore, Lonp1 most resembles the mitochondrial pyruvate dehydrogenase complex (PDC); a complex of three enzymes catalysing the conversion of pyruvate into Acetyl-CoA, which has been shown to translocate from the mitochondrial matrix to the nucleus during cell cycle progression, in response to growth signals, such as serum or epidermal growth factor.

We have also established that Lonp1 can localize in the nucleus in response to HS. Our proposal of a link between Lonp1 and HS is not without precedent, as several works have already demonstrated a complex interplay between them in prokaryotes. Lonp1 is highly conserved throughout evolution, and was first described in E. coli, where it is known as La protease. Its roles in this species are multiple, spanning from protein quality control, radiation resistance, filamentation, capsular polysaccharide production, cell division, and survival under starvation conditions (5). Over the past decade, a role for bacterial Lon in proteotoxic stress response has also been described (36-38). For example, in Caulobacter crescentus, the proteotoxic stress induces cell cycle arrest by triggering the degradation of the replication initiator DnaA by Lon (37). In Yersinia pestis, HS protein Q (hspQ), which is induced by HS, regulates the substrate preference and catalytic efficiency of Lon (38). Thus, our study demonstrates that this link is maintained in eukaryotes, and particularly in mammalian cells. In this study, we report the translocation of Lonp1 to the nucleus when cells are exposed to HS, but not to other stress stimuli, such as genotoxic stress or cell cycle block. Previous reports have indicated that Lonp1 is stress-responsive and is upregulated in the presence of HS (39); our observation further expands the importance of Lonp1 as a stress response protein, and its range of actions in the human cell. 
It is theoretically possible that the nuclear increase of Lonp1 in the presence of HS could be caused by the direct import of the neo-synthesized protein from the cytoplasm into the nucleus. However, we tended to exclude these possibilities, for several reasons. Data we obtained on cells overexpressing eGFP-ISO1 indicated that HS determines an increase of the protein level in the nucleus, also for the eGFP tagged form. As HS does not modify the transcription of the plasmid-encoded protein, the relative increase in the nuclear fraction form can only be due to a relocation of the mitochondrial form. Furthermore, the MW of the protein present in the nuclear fraction is identical to that present in the mitochondria, suggesting that MTS is cut away from the enzyme when it relocates into the nucleus. The way Lonp1 is exported from mitochondria to the nucleus is still unknown.

An ongoing open question is the nature of the interaction between Lonp1 and HSF1. As Lonp1 displays a proteolytic activity, we hypothesize that Lonp1 can contact HSF1 to degrade it, thereby modulating HSR. This hypothesis is supported by our observation that under HS conditions, Lonp1 knock down determines a higher expression of HSF1 target genes.

We also showed that three splicing variants of Lonp1 can be detected in human cells, which encode for three isoforms differing for their $\mathrm{N}$-terminus sequence. These three isoforms display different subcellular distributions, and their expression varies significantly among cell types, and between normal and transformed cells. ISO2 is the form expressed at higher transcriptional levels in many cell types and, more importantly, is the form whose expression increases more in cancer tissues in comparison to healthy tissue. This observation has important consequences in the evaluation of data concerning Lonp1 expression in cancer previously reported. Several authors, including us, have shown an increase in Lonp1 expression in different cancer types (8, 40-42). However, these data clearly indicate that only one of the isoforms increases, and the up-regulated isoform changes, depending on the type of cancer. Therefore, in future studies it will be important 
to clearly identify which isoform is analysed/observed when correlations between Lonp1 levels and clinical data are reported.

The biological significance of the ISO3 remains to be determined. The observation that its expression is barely detectable in many cell types strongly suggests that its function(s), if any, is not crucial for cell homeostasis. Our confocal analysis has shown a distribution that is clearly non-mitochondrial, without any overlap with ISO1 and ISO2. Thus, it is unlikely that heterooligomer ISO1/ISO3 or ISO2/ISO3 can form in cells.

The N-term region of Lonp1, which is shorter in ISO3, has been shown to be crucial for oligomerization, and for substrate specificity, and in vitro studies have shown that the lack of the first 270 aa impairs proteolytic activity (43). Further studies on the purified ISO-3, which is 74 aa longer than that analysed by Kereiche et al., could clarify if its proteolytic activity is preserved, if the hexameric structure is properly maintained, and if the lack of aa 1-196 leads to a change in the substrate specificity of the enzyme.

Mutations of LONP1 gene are associated with Cerebral Ocular Dental Auricular Skeletal Anomalies Syndrome (CODAS), a complex multisystemic and developmental disorder $(5,10)$. The pathogenetic link between LONP1 mutations and the signs and symptoms of the disease are still elusive. It could be of great interest to understand if the Lonp1 mutants observed in CODAS patients display an aberrant nuclear localization, and if this difference can contribute to the development of the anomalies observed in CODAS patients.

\section{Conclusion}

This study observed the detection of Lonp1 in the nucleus in response to HS where is modulates the cellular response through its interaction with HSF1. All three isoforms were found to relocate to the nucleus. These findings highlight a novel and surprising new role of 
Lonp1 in cellular homeostasis, expanding the growing list of mitochondrial proteins that play a role in the nucleus.

\section{Declarations}

\section{Funding}

This work was supported by the "Associazione Italiana Ricerca sul Cancro" (AIRC, Investigator grant number 19786 to MP), and by a grant from Associazione Angela Serra per la Ricerca sul Cancro (Modena) to AC.

\section{Conflict of interests}

The authors declare that they have no conflict of interest.

\section{Availability of data and material}

RNA-seq data from this study have been deposited at Gene Expression Omnibus database (GEO, https://www.ncbi.nlm.nih.gov/geo/) with accession number GSE164834.

\section{Authors' contributions}

L.G.: conceptualization, investigation, data curation, formal analysis, manuscript writing; R.B.: investigation, formal analysis; A.D.G.: investigation, formal analysis; G.C. investigation, formal analysis; D.L.T.: formal analysis; F.B.: investigation, formal analysis; L.L. investigation; S.D.B.: investigation, formal analysis; G.Z, M.F: bioinformatic analysis; M.N.: data analysis, A.C: manuscript writing and revision; M.P.: conceptualization, supervision, data curation, formal analysis, manuscript writing. 


\section{Acknowledgements}

Sara De Biasi and Lara Gibellini are International Society for Advancement in Cytometry (ISAC) Marylou Ingram Scholars. We gratefully acknowledge the "Associazione Italiana Mogli Medici" in Modena for the kind donation of the Trans-Blot Turbo Blotting System to AC. Dr. Paola Paglia (ThermoFisher) is acknowledged for her technical support. 


\section{LEGEND TO FIGURES}

Figure 1. Lonp1 localizes in the nuclei of mouse colon tissue and human colon tissue.

A. Representative images of Lonp1 IHC on murine colon tissue, showing Lonp1 nuclear localization. B. Representative images of Lonp1 IF on murine colon tissue, showing Lonp1 nuclear localization. Nuclei were counterstained with DAPI. C. Representative Lonp1 IHC staining in intestinal crypt. Cells of the crypt were sectioned into three zones: the lower (0 to $+4)$, upper $(+5$ to +15$)$, and non $(>+15)$-crypt zones. The histogram represents the percentage of cells with nuclear Lonp1 per crypt zone ( $\mathrm{n}=10$ crypts per group). Data represent the mean \pm SD. D. Alignment of Lonp1 protein sequences (amino acids 218 to 256) from the indicated mammalian species. HS, Homo sapiens; CL, Canis lupus familiaris; MM, Mus musculus; RN, Rattus norvegicus. The nuclear targeting sequence is highlighted in red. E. Representative images of Lonp1 IHC on human colon tissue, showing Lonp1 nuclear localization (arrows). Bar: $200 \mu \mathrm{m}$.

\section{Figure 2. A fraction of Lonp1 localizes in the nuclei of human cells}

A. Representative confocal microscopy images of SW620 cells after immunostaining with anti-Lonp1 and anti-hMit Abs. Nuclei were counterstained with DAPI. B. Representative immunoblot of cytosolic (C) and nuclear (N) fractions obtained from SW620 cells. Lamin B1 is the nuclear fraction loading control, whereas $\beta$-actin is the cytosolic fraction loading control. Immunoblots of Sirtuin-3 (SIRT3) and TOM20 are also reported to indicate that mitochondrial contamination is not present. C. Quantification of the ratio of nuclear and cytosolic Lonp1 ( $n=3$ independent experiments). $P<0.05$. D. Representative confocal microscopy images of SW620 cells after immunostaining with anti-Lonp1 antibody. Cells were treated or not with leptomycin B (LMB) to inhibit nuclear export of proteins. Staining of p62 was reported as positive control for LBM treatment. Nuclei were counterstained with 
DAPI. E. Representative immunoblot of cytosolic (cytosol) and nuclear (N) fractions obtained from SW620 cells, treated or not with LBM. Lamin B1 is the nuclear fraction loading control, whereas $\beta$-actin is the cytosolic fraction loading control. Immunoblot for p62 is also reported as positive control for LMB treatment.

\section{Figure 3. DNA damage or cell cycle do not influence Lonp1 nuclear expression}

A. Cell cycle analysis of SW620 cells treated or not with thymidine, using 5-ethynyl-2'deoxyuridine. Cell were treated with thymidine (Thy) to block cell cycle in the G1 phase. Representative immunoblot of cytosolic $(\mathrm{C})$ and nuclear $(\mathrm{N})$ fractions obtained from SW620 cells, treated or not with thymidine. B. Representative confocal microscopy images of SW620 control cells (CTR), and cells irradiated with 5 Gy (IR), immunostained with anti- $\gamma$ $\mathrm{H} 2 \mathrm{AX}$. Bars: $10 \mu \mathrm{m}$. Representative immunoblot reporting the expression of $\gamma-\mathrm{H} 2 \mathrm{AX}$ in SW620 control cells, and cells irradiated with 5 Gy. C. Representative confocal microscopy images of SW620 control cells (CTR), and cells treated with hydroxyurea (HU) or doxorubicin (DOXO), immunostained with anti- $\gamma-\mathrm{H} 2 \mathrm{AX}$. Bars: $10 \mu \mathrm{m}$. Representative immunoblot of SW620 cells (CTRL), and cells treated with increasing concentrations of HU and DOXO. Right panel: Representative immunoblot of total lysate (TL), cytosolic (cyt) and nuclear $(\mathrm{N})$ fractions obtained from SW620 treated or not with $4 \mathrm{mM} \mathrm{HU}$ or $2 \mu \mathrm{M}$ DOXO.

Figure 4. Lonp1 relocates in the nucleus in response to heat shock.

A. Representative confocal microscopy images of SW620 cells after immunostaining with anti-Lonp1 antibody. Cells were heat-shocked (HS) at $42^{\circ} \mathrm{C}$ and then left to recover (HS+REC). B. Representative immunoblot showing Lonp1 expression in cytosolic and nuclear fractions from SW620 cells maintained at $37^{\circ} \mathrm{C}(\mathrm{CTR})$, at $42^{\circ} \mathrm{C}$ for $3 \mathrm{hrs}$ to induce heat shock (HS) and kept at $42^{\circ} \mathrm{C}$ for $3 \mathrm{hrs}$ and then left for 1 hour at $37^{\circ} \mathrm{C}$ to recover (HS+REC). HSP70 was used as a positive control for HS treatment, and Lamin B1 and actin 
as markers for nuclear and cytosolic fractions, respectively. C. Histogram representing the relative levels of Lonp1 in nuclear fractions, obtained from three independent experiments. Data are shown as mean SD. ${ }^{*}=p<0.05$. D. Representative immunoprecipitation experiment showing the interaction between Lonp1-eGFP and HSF1 in SW620 cells after heat-shock (HS) and then left 3 hours at $37^{\circ} \mathrm{C}$ to recover (HS+REC). Lysates were immunoprecipitated with anti-eGFP and immunoblotted with anti-HSF1. E. Representative immunoprecipitation experiment showing the interaction between Lonp1-eGFP and HSF1 in SW620 cells after heat-shock (HS) and then left 3 hours at $37^{\circ} \mathrm{C}$ to recover (HS+REC). Lysates were immunoprecipitated with anti-HSF1 and immunoblotted with anti-eGFP. F. Representative immunoblot showing the quantification of HSF1 in SW620 cells transfected with scramble small-interfering RNAs (siCTRL) and cells transfected with small-interfering RNAs against Lonp1 (siLonp1 and siLonp2), after HS and HS+REC. G. Quantification of the mRNA levels of HSP70 in SW620 cells transfected with scramble small-interfering RNAs (siCTRL) and cells transfected with small-interfering RNAs against Lonp1 (siLonp1), after HS and HS+REC. ${ }^{*} \mathrm{P}<0.05$.

\section{Figure 5. Silencing of Lonp1 enhances HSF1-mediated response to HS.}

A. Number of genes significantly up- or down-regulated in SW620 cells transfected with small-interfering RNAs against Lonp1 (siLonp1) or with a scramble siRNA, kept at $37^{\circ} \mathrm{C}$ (Ctrl) or at $42^{\circ}$ for $1 \mathrm{~h}(\mathrm{HS})$. FDR q value $\leq 0.05$ was used to identify differentially expressed genes, with fold change $(F C)>2$ for up-regulated and $F C<2$ for down-regulated genes. B) Venn diagram showing the overlap between genes upregulated in HS, in cells where Lonp1 was silenced (siLonp1) or not (Scramble) in SW620 cells. C. Left panel: Gene set enrichment analysis (GSEA) plots for the "Reactome - HSF1 dependent transactivation" signature in SW620 cells transfected with small-interfering RNA against Lonp1 (siLonp1) in comparison with a scramble siRNA, kept at $37^{\circ} \mathrm{C}$. Right panel: Gene set enrichment 
analysis (GSEA) plots for the "Reactome - HSF1 dependent transactivation" signature in SW620 cells transfected with small-interfering RNA against Lonp1 (siLonp1) in comparison with a scramble siRNA, kept at $42^{\circ}$ for $1 \mathrm{~h}$. The Normalized enrichment score (NES) and FDR values for both analyses are reported.

Figure 6. Human cells express three isoforms of Lonp1, whose subcellular localization is different.

A. Diagram depicting the location of mitochondrial targeting sequence (MTS), of the nuclear localization signal (NLS), and of the $N, A A A+$ and $P$ domains of three isoforms of human Lonp1. The length of the three isoforms is indicated. B. Histogram showing the relative expression levels of mRNAs encoding Lonp1 isoform-1 (ISO1), Lonp1 isoform-2 (ISO2), Lonp1 isoform-3 (ISO3) in normal tissue, obtained from TGCA Splicing Variant database (TSVdb). C. Representative immunoblot detecting Lonp1 using anti eGFP (left panel) and anti Lonp1 (right panel) Abs in cells transfected with Lonp1 ISO-eGFP, ISO2-eGFP or ISO3eGFP. The position of the bands corresponding to the exogenous, eGFP-tagged form and the endogenous form of Lonp1 is indicated. D. Representative immunoblots showing Lonp1 expression in cytosolic $(\mathrm{C})$ and nuclear $(\mathrm{N})$ fractions (left panel) and cytosolic $(\mathrm{C})$ and mitochondrial (M) fractions (right panel) in SW620 cells transfected with constructs bearing Lonp1 isoforms tagged at C-term with enhanced green fluorescent protein (eGFP). Lonp1 has been detected using anti-eGFP antibody. E. Representative confocal microscopy images of HeLa cells transfected with three different constructs bearing Lonp1 isoforms tagged at C-term with enhanced green fluorescent protein (eGFP), namely Lonp1-ISO1, Lonp1-ISO2 or Lonp1-ISO3. Mitochondria were stained with anti-hMit and nuclei were counterstained with DAPI. 


\section{Figure 7. Lonp1 ISO1 increases in the nucleus in response to heat shock}

A. Representative immunoblot detecting Lonp1 in cells transfected with Lonp1 ISO1-eGFP, ISO2 -eGFP or ISO3-eGFP in untreated conditions (CTR), undergoing HS for 3 hours (HS) and HS for 3 hours followed by recovery (HS+REC). B. Histograms showing the relative expression levels of Lonp1 ISO1, ISO2, and ISO3 in in untreated cells (CTR), cells undergoing HS for 3 hours (HS) and HS for 3 hours followed by recovery (HS+REC). Data are reported as mean $\pm S D$ of three independent experiments. C. Representative confocal microscopy images of SW620 cells transfected with Lonp1 ISO1-eGFP, ISO2 -eGFP or ISO3-eGFP, in untreated conditions (CTR), undergoing HS and HS followed by recovery (HS+REC). D. Histogram showing the relative expression levels of Lonp1 ISO1 mRNA in the indicated primary solid tumours $(\mathrm{T})$ and the normal $(\mathrm{N})$ counterparts, as obtained from TGCA Splicing Variant database (TSVdb). Rectum: rectum adenocarcinoma; Colon: colon adenocarcinoma; Lung: lung adenocarcinoma; Cervical: cervical squamous cell carcinoma and endocervical carcinoma; Bladder: bladder urothelial carcinoma; Prostate: prostate adenocarcinoma; Breast: breast invasive carcinoma; Head and neck: head and neck squamous cell carcinoma; Renal: renal clear cell carcinoma. Data are in log scale; mean, 24 and 75 percentile, max and min values are shown. ${ }^{*}=p<0.05 ;{ }^{* *}=p<0.01 ;{ }^{* * *}=p<0.0001$. E. Histogram showing the relative expression levels of Lonp1 ISO2 mRNA in the indicated primary solid tumours $(\mathrm{T})$ and the normal $(\mathrm{N})$ counterparts, as obtained from TGCA Splicing Variant database (TSVdb). Rectum: rectum adenocarcinoma; Colon: colon adenocarcinoma; Lung: lung adenocarcinoma; Cervical: cervical squamous cell carcinoma and endocervical carcinoma; Bladder: bladder urothelial carcinoma; Prostate: prostate adenocarcinoma; Breast: breast invasive carcinoma; Head and neck: head and neck squamous cell carcinoma; Renal: renal clear cell carcinoma. Data are in log scale; mean, 24 and 75 percentile, max and min values are shown. ${ }^{*}=p<0.05 ;{ }^{* *}=p<0.01 ;{ }^{* * *}=p<0.0001$. 


\section{Supplementary Figure 1}

Representative confocal microscopy images of SW48, SW480 and HeLa cells after immunostaining with anti-Lonp1 and anti-hMit antibodies. Nuclei were counterstained with DAPI.

\section{Supplementary Figure 2}

Principal component analysis and unsupervised cluster analysis and of RNAseq data from SW620 cells undergoing heat shock for 1 hour, heat shock for $1 \mathrm{~h}$ followed by $1 \mathrm{~h}$ hour of recovery, and cells kept at $37^{\circ} \mathrm{C}$, when Lonp1 was silenced or not. Ctrl siScramble: cells transfected with a scrambled siRNA, kept at $37^{\circ} \mathrm{C}$. Ctrl siLonp1: cells transfected with siRNA for LONP1, kept at $37^{\circ} \mathrm{C}$. HS siScramble: cells transfected with scrambled siRNA, kept at $42^{\circ} \mathrm{C}$ for 1 hour. HS_siLONP1: cells transfected with LONP1 siRNA, kept at $42^{\circ} \mathrm{C}$ for 1 hour. Hs +REC siScramble: cells transfected with scrambled siRNA, kept at $42^{\circ} \mathrm{C}$ for 1 hour and left at $37^{\circ} \mathrm{C}$ for 1 hour to recover. HS + Rec siLonp1: cells transfected with LONP1 siRNA, kept at $42^{\circ} \mathrm{C}$ for 1 hour and left at $37^{\circ} \mathrm{C}$ for 1 hour to recover.

\section{Supplementary Figure 3}

Representative confocal microscopy images of HeLa co-transfected with constructs bearing Lonp1 isoforms tagged with enhanced green fluorescent protein (eGFP, in green) or mCherry (in red). Nuclei were counterstained with DAPI.

\section{Supplementary Figure 4}

Histogram showing the levels of Lonp1 isoform-3 (ISO3) in normal tissues $(\mathrm{N})$ or primary solid tumors $(T)$ obtained from TSVdb. Data represent the mean \pm SD. Rectum: rectum adenocarcinoma; Colon: colon adenocarcinoma; Lung: lung adenocarcinoma; Cervical: 
cervical squamous cell carcinoma and endocervical carcinoma; Bladder: bladder urothelial carcinoma; Prostate: prostate adenocarcinoma; Breast: breast invasive carcinoma; Head and neck: head and neck squamous cell carcinoma; Renal: renal clear cell carcinoma. $\mathrm{P}<0.05$.

Supplementary table 1. Gene Ontology (GO) terms as determined by DAVID in cells under HS in comparison to cells kept at $37^{\circ} \mathrm{C}$, either with silenced Lonp1 (SiLonp1) or in cells treated with a scramble siRNA (Scramble). Significantly enriched terms (BenjaminiHochberg adjusted $p$ value $\leq 0.05$ ) are in bold.

Supplementary table 2. Reactome pathways found to be enriched in cells under HS in comparison to cells kept at $37^{\circ} \mathrm{C}$, either with silenced Lonp1 (SiLonp1) or in cells treated with a scramble siRNA (siScramble). Significantly enriched pathways (adjusted $p$ value $\leq$ 0.05) are in bold.

Supplementary table 3 . List of the first 25 genes target of HSF1, upregulated in SW620 cells under HS. The FC with respect to control cells, kept at $37^{\circ} \mathrm{C}$, is shown for cells treated with siLonp1 or with a scrambled siRNA. The FC ratio between is also shown.

Supplementary table 4. Enriched gene sets from the Reactome collection in SW620 cells when Lonp1 is silenced, in comparison to scramble siRNA, either at $37^{\circ} \mathrm{C}(\mathrm{Ctrl})$ and at $42^{\circ} \mathrm{C}$ for 1 hour (HS), as detected with GSEA. Data are ranked based on Normalized enrichment score (NES). 


\section{References}

1. Monaghan RM and Whitmarsh AJ (2015) Mitochondrial Proteins Moonlighting in the Nucleus. Trends Biochem Sci 40(12): 728-735.

2. Monaghan RM, Barnes RG, Fisher K, Andreou T, Rooney N, Poulin GB, and Whitmarsh AJ (2015) A nuclear role for the respiratory enzyme CLK-1 in regulating mitochondrial stress responses and longevity. Nat Cell Biol 17(6): 782-92.

3. Sutendra G, Kinnaird A, Dromparis P, Paulin R, Stenson TH, Haromy A, Hashimoto K, Zhang N, Flaim E, and Michelakis ED (2014) A nuclear pyruvate dehydrogenase complex is important for the generation of acetyl-CoA and histone acetylation. Cell 158(1): 84-97.

4. Yogev O, Yogev O, Singer E, Shaulian E, Goldberg M, Fox TD, and Pines O (2010) Fumarase: a mitochondrial metabolic enzyme and a cytosolic/nuclear component of the DNA damage response. PLoS Biol 8(3): e1000328.

5. Pinti M, Gibellini L, Liu Y, Xu S, Lu B, and Cossarizza A (2015) Mitochondrial Lon protease at the crossroads of oxidative stress, ageing and cancer. Cell Mol Life Sci 72(24): 4807-24.

6. Gibellini L, Pinti M, Boraldi F, Giorgio V, Bernardi P, Bartolomeo R, Nasi M, De Biasi S, Missiroli S, Carnevale G, et al (2014) Silencing of mitochondrial Lon protease deeply impairs mitochondrial proteome and function in colon cancer cells. FASEB J 28(12): 5122-35.

7. Quiros PM, Espanol Y, Acin-Perez R, Rodriguez F, Barcena C, Watanabe K, Calvo E, Loureiro M, Fernandez-Garcia MS, Fueyo A, et al (2014) ATP-dependent Lon protease controls tumor bioenergetics by reprogramming mitochondrial activity. Cell Rep 8(2): 542-56.

8. Gibellini L, Losi L, De Biasi S, Nasi M, Lo Tartaro D, Pecorini S, Patergnani S, Pinton P, De Gaetano A, Carnevale G, et al (2018) LonP1 Differently Modulates Mitochondrial Function and Bioenergetics of Primary Versus Metastatic Colon Cancer Cells. Front Oncol 8: 254. 
9. Cheng CW, Kuo CY, Fan CC, Fang WC, Jiang SS, Lo YK, Wang TY, Kao MC, and Lee AY (2013) Overexpression of Lon contributes to survival and aggressive phenotype of cancer cells through mitochondrial complex I-mediated generation of reactive oxygen species. Cell Death Dis 4: e681.

10. Pinti M, Gibellini L, Nasi M, De Biasi S, Bortolotti CA, lannone A, and Cossarizza A (2016) Emerging role of Lon protease as a master regulator of mitochondrial functions. Biochim Biophys Acta 1857(8): 1300-1306.

11. Strauss KA, Jinks RN, Puffenberger EG, Venkatesh S, Singh K, Cheng I, Mikita N, Thilagavathi J, Lee J, Sarafianos S, et al (2015) CODAS syndrome is associated with mutations of LONP1, encoding mitochondrial AAA+ Lon protease. Am J Hum Genet 96(1): 121-35.

12. Bota DA and Davies KJ (2002) Lon protease preferentially degrades oxidized mitochondrial aconitase by an ATP-stimulated mechanism. Nat Cell Biol 4(9): 674-80.

13. Lu B, Yadav S, Shah PG, Liu T, Tian B, Pukszta S, Villaluna N, Kutejova E, Newlon CS, Santos $\mathrm{JH}$, et al (2007) Roles for the human ATP-dependent Lon protease in mitochondrial DNA maintenance. J Biol Chem 282(24): 17363-74.

14. Ngo JK and Davies KJ (2007) Importance of the lon protease in mitochondrial maintenance and the significance of declining lon in aging. Ann N Y Acad Sci 1119: 78-87.

15. Polo M, Alegre F, Moragrega AB, Gibellini L, Marti-Rodrigo A, Blas-Garcia A, Esplugues JV, and Apostolova N (2017) Lon protease: a novel mitochondrial matrix protein in the interconnection between drug-induced mitochondrial dysfunction and endoplasmic reticulum stress. Br J Pharmacol 174(23): 4409-4429.

16. De Gaetano A, Gibellini L, Bianchini E, Borella R, De Biasi S, Nasi M, Boraldi F, Cossarizza A, and Pinti M (2020) Impaired Mitochondrial Morphology and Functionality in Lonp1(wt/-) Mice. J Clin Med 9(6). 
17. Maraldi T, Beretti F, Anselmi L, Franchin C, Arrigoni G, Braglia L, Mandrioli J, Vinceti M, and Marmiroli S (2019) Influence of selenium on the emergence of neuro tubule defects in a neuron-like cell line and its implications for amyotrophic lateral sclerosis. Neurotoxicology 75: 209-220.

18. Cossarizza A, Chang HD, Radbruch A, Acs A, Adam D, Adam-Klages S, Agace WW, Aghaeepour $N$, Akdis M, Allez M, et al (2019) Guidelines for the use of flow cytometry and cell sorting in immunological studies (second edition). Eur J Immunol 49(10): 1457-1973.

19. Dobin A, Davis CA, Schlesinger F, Drenkow J, Zaleski C, Jha S, Batut P, Chaisson M, and Gingeras TR (2013) STAR: ultrafast universal RNA-seq aligner. Bioinformatics 29(1): 15-21.

20. Liao Y, Smyth GK, and Shi W (2014) featureCounts: an efficient general purpose program for assigning sequence reads to genomic features. Bioinformatics 30(7): 923-30.

21. Robinson MD, McCarthy DJ, and Smyth GK (2010) edgeR: a Bioconductor package for differential expression analysis of digital gene expression data. Bioinformatics 26(1): 139-40.

22. Chen EY, Tan CM, Kou Y, Duan Q, Wang Z, Meirelles GV, Clark NR, and Ma'ayan A (2013) Enrichr: interactive and collaborative HTML5 gene list enrichment analysis tool. BMC Bioinformatics 14: 128.

23. Huang da W, Sherman BT, and Lempicki RA (2009) Systematic and integrative analysis of large gene lists using DAVID bioinformatics resources. Nat Protoc 4(1): 44-57.

24. Huang da W, Sherman BT, and Lempicki RA (2009) Bioinformatics enrichment tools: paths toward the comprehensive functional analysis of large gene lists. Nucleic Acids Res 37(1): 113.

25. Kuleshov MV, Jones MR, Rouillard AD, Fernandez NF, Duan Q, Wang Z, Koplev S, Jenkins SL, Jagodnik KM, Lachmann A, et al (2016) Enrichr: a comprehensive gene set enrichment analysis web server 2016 update. Nucleic Acids Res 44(W1): W90-7. 
26. Subramanian A, Tamayo P, Mootha VK, Mukherjee S, Ebert BL, Gillette MA, Paulovich A, Pomeroy SL, Golub TR, Lander ES, et al (2005) Gene set enrichment analysis: a knowledgebased approach for interpreting genome-wide expression profiles. Proc Natl Acad Sci U S A 102(43): 15545-50

27. Liberzon A, Birger C, Thorvaldsdottir H, Ghandi M, Mesirov JP, and Tamayo P (2015) The Molecular Signatures Database (MSigDB) hallmark gene set collection. Cell Syst 1(6): 417425

28. Gibellini L, De Gaetano A, Mandrioli M, Van Tongeren E, Bortolotti CA, Cossarizza A, and Pinti M (2020) The biology of Lonp1: More than a mitochondrial protease. Int Rev Cell Mol Biol 354: 1-61

29. Matsushima Y, Goto Y, and Kaguni LS (2010) Mitochondrial Lon protease regulates mitochondrial DNA copy number and transcription by selective degradation of mitochondrial transcription factor A (TFAM). Proc Natl Acad Sci U S A 107(43): 18410-5

30. Kovacs D, Sigmond T, Hotzi B, Bohar B, Fazekas D, Deak V, Vellai T, and Barna J (2019) HSF1Base: A Comprehensive Database of HSF1 (Heat Shock Factor 1) Target Genes. Int J Mol Sci 20(22):

31. El Marabti E and Younis I (2018) The Cancer Spliceome: Reprograming of Alternative Splicing in Cancer. Front Mol Biosci 5: 80

32. Gibellini L, Pinti M, Bartolomeo R, De Biasi S, Cormio A, Musicco C, Carnevale G, Pecorini S, Nasi M, De Pol A, et al (2015) Inhibition of Lon protease by triterpenoids alters mitochondria and is associated to cell death in human cancer cells. Oncotarget 6(28): 25466-83

33. Pareek G, Thomas RE, Vincow ES, Morris DR, and Pallanck LJ (2018) Lon protease inactivation in Drosophila causes unfolded protein stress and inhibition of mitochondrial translation. Cell Death Discov 4: 51 
34. Horner SM, Wilkins C, Badil S, Iskarpatyoti J, and Gale M, Jr. (2015) Proteomic analysis of mitochondrial-associated ER membranes (MAM) during RNA virus infection reveals dynamic changes in protein and organelle trafficking. PLoS One 10(3): e0117963

35. Poston CN, Krishnan SC, and Bazemore-Walker CR (2013) In-depth proteomic analysis of mammalian mitochondria-associated membranes (MAM). J Proteomics 79: 219-30

36. Bhattacharjee S, Dasgupta R, and Bagchi A (2017) Elucidation of the molecular mechanism of heat shock proteins and its correlation with $\mathrm{K} 722 \mathrm{Q}$ mutations in Lon protease. Biosystems 159: $12-22$

37. Jonas K, Liu J, Chien P, and Laub MT (2013) Proteotoxic stress induces a cell-cycle arrest by stimulating Lon to degrade the replication initiator DnaA. Cell 154(3): 623-36

38. Puri N and Karzai AW (2017) HspQ Functions as a Unique Specificity-Enhancing Factor for the AAA+ Lon Protease. Mol Cell 66(5): 672-683 e4

39. Ngo JK and Davies KJ (2009) Mitochondrial Lon protease is a human stress protein. Free Radic Biol Med 46(8): 1042-8

40. Bernstein SH, Venkatesh S, Li M, Lee J, Lu B, Hilchey SP, Morse KM, Metcalfe HM, Skalska J, Andreeff M, et al (2012) The mitochondrial ATP-dependent Lon protease: a novel target in lymphoma death mediated by the synthetic triterpenoid CDDO and its derivatives. Blood 119(14): 3321-9

41. Liu Y, Dong H, Peng X, Gao Q, Jiang H, Xu G, Qin Y, Niu J, Sun S, Li P, et al (2019) RNA-seq reveals the critical role of Lon protease in stress response and Brucella virulence. Microb Pathog 130: 112-119

42. Liu Y, Lan L, Huang K, Wang R, Xu C, Shi Y, Wu X, Wu Z, Zhang J, Chen L, et al (2014) Inhibition of Lon blocks cell proliferation, enhances chemosensitivity by promoting apoptosis and 
decreases cellular bioenergetics of bladder cancer: potential roles of Lon as a prognostic marker and therapeutic target in baldder cancer. Oncotarget 5(22): 11209-24

43. Kereiche S, Kovacik L, Bednar J, Pevala V, Kunova N, Ondrovicova G, Bauer J, Ambro L, Bellova J, Kutejova E, et al (2016) The N-terminal domain plays a crucial role in the structure of a fulllength human mitochondrial Lon protease. Sci Rep 6: 33631 
Figures
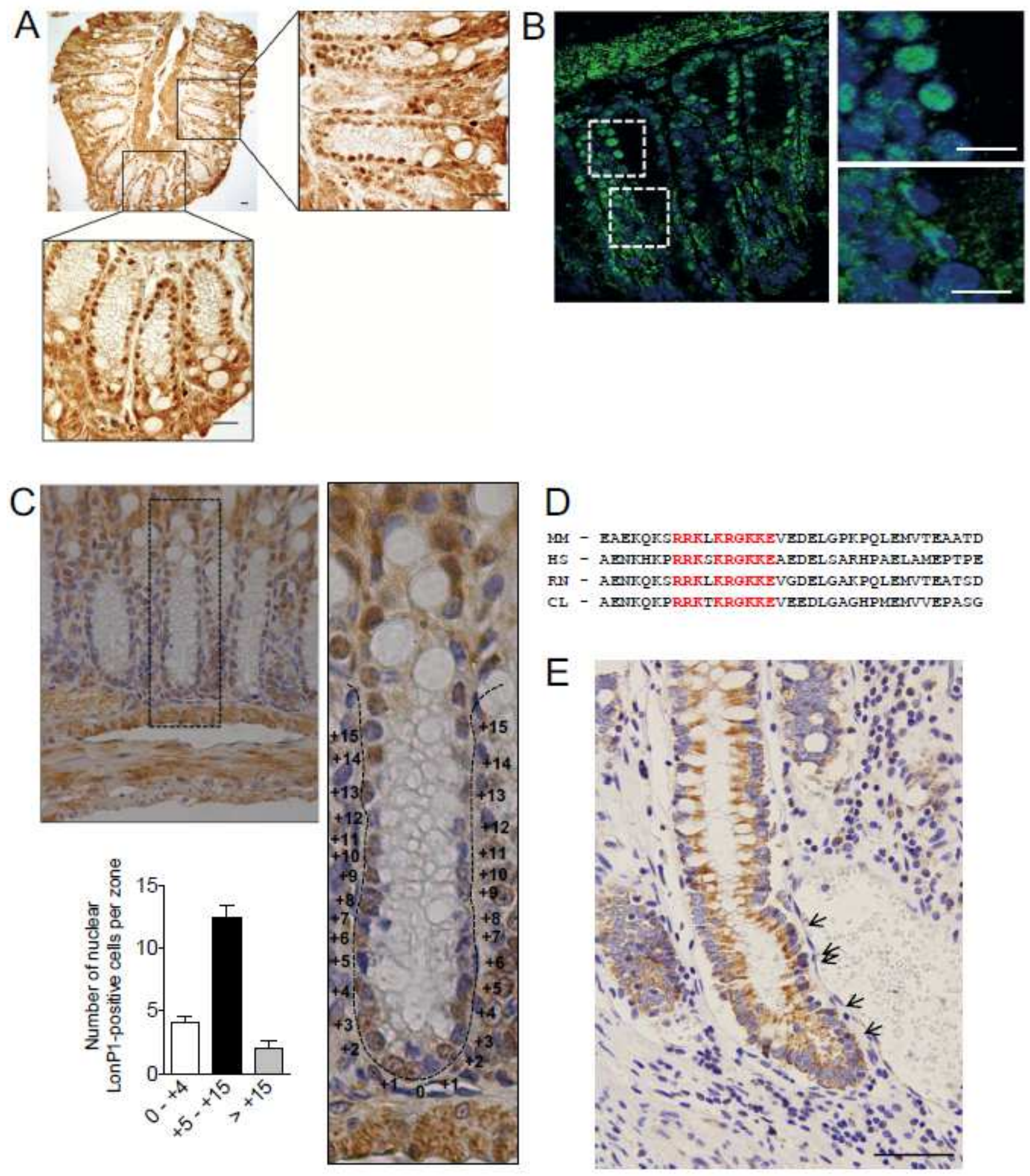

\section{Figure 1}

Lonp1 localizes in the nuclei of mouse colon tissue and human colon tissue. A. Representative images of Lonp1 IHC on murine colon tissue, showing Lonp1 nuclear localization. B. Representative images of Lonp1 IF on murine colon tissue, showing Lonp1 nuclear localization. Nuclei were counterstained with 
DAPI. C. Representative Lonp1 IHC staining in intestinal crypt. Cells of the crypt were sectioned into three zones: the lower $(0$ to +4$)$, upper $(+5$ to +15$)$, and non $(>+15)$-crypt zones. The histogram represents the percentage of cells with nuclear Lonp1 per crypt zone ( $n=10$ crypts per group). Data represent the mean \pm SD. D. Alignment of Lonp1 protein sequences (amino acids 218 to 256) from the indicated mammalian species. HS, Homo sapiens; CL, Canis lupus familiaris; MM, Mus musculus; RN, Rattus norvegicus. The nuclear targeting sequence is highlighted in red. E. Representative images of Lonp1 IHC on human colon tissue, showing Lonp1 nuclear localization (arrows). Bar: $200 \mu \mathrm{m}$.
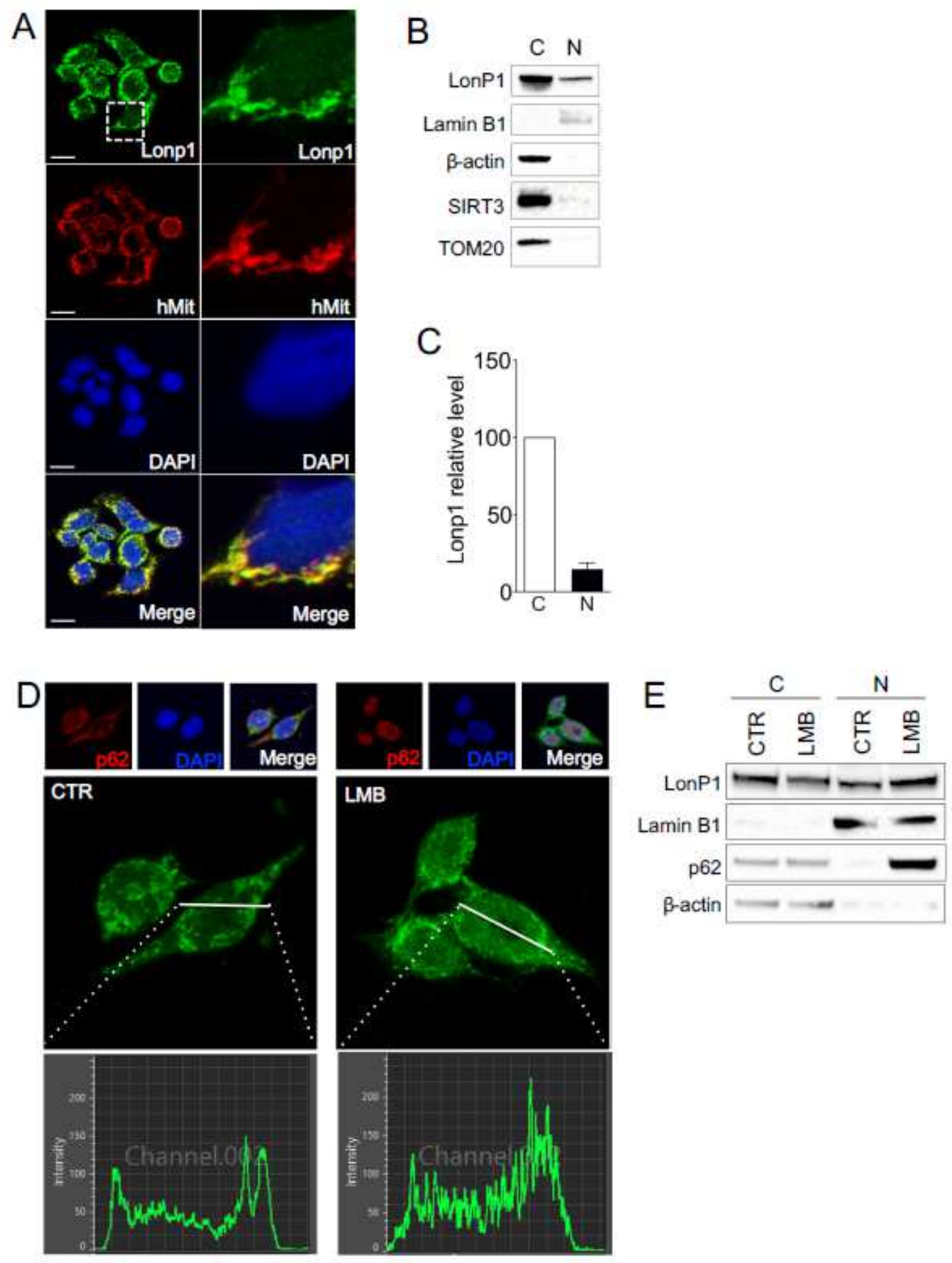


\section{Figure 2}

A fraction of Lonp1 localizes in the nuclei of human cells A. Representative confocal microscopy images of SW620 cells after immunostaining with anti-Lonp1 and anti-hMit Abs. Nuclei were counterstained with DAPI. B. Representative immunoblot of cytosolic (C) and nuclear (N) fractions obtained from SW620 cells. Lamin B1 is the nuclear fraction loading control, whereas b-actin is the cytosolic fraction loading control. Immunoblots of Sirtuin-3 (SIRT3) and TOM20 are also reported to indicate that mitochondrial contamination is not present. C. Quantification of the ratio of nuclear and cytosolic Lonp1 $(n=3$ independent experiments). P<0.05. D. Representative confocal microscopy images of SW620 cells after immunostaining with anti-Lonp1 antibody. Cells were treated or not with leptomycin B (LMB) to inhibit nuclear export of proteins. Staining of p62 was reported as positive control for LBM treatment. Nuclei were counterstained with DAPI. E. Representative immunoblot of cytosolic (cytosol) and nuclear (N) fractions obtained from SW620 cells, treated or not with LBM. Lamin B1 is the nuclear fraction loading control, whereas b-actin is the cytosolic fraction loading control. Immunoblot for p62 is also reported as positive control for LMB treatment. 

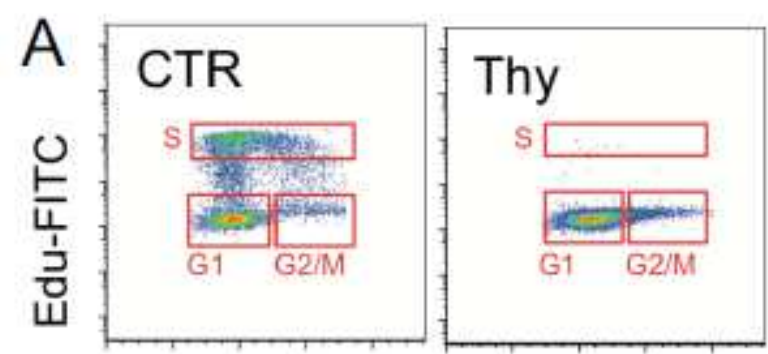

FxCycle
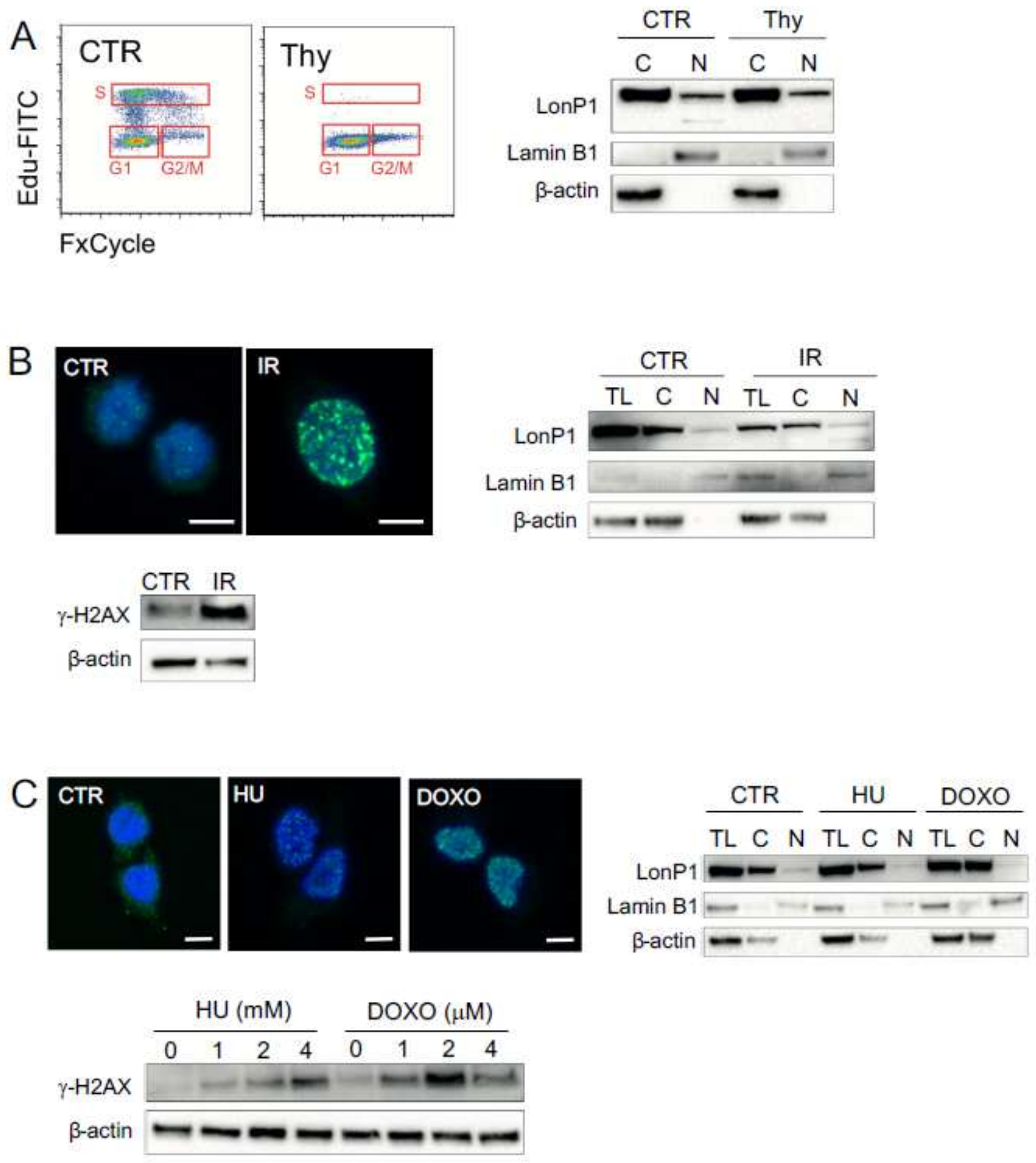

\section{Figure 3}

DNA damage or cell cycle do not influence Lonp1 nuclear expression A. Cell cycle analysis of SW620 cells treated or not with thymidine, using 5-ethynyl-2' deoxyuridine. Cell were treated with thymidine (Thy) to block cell cycle in the $\mathrm{G} 1$ phase. Representative immunoblot of cytosolic (C) and nuclear (N) fractions obtained from SW620 cells, treated or not with thymidine. B. Representative confocal microscopy images of SW620 control cells (CTR), and cells irradiated with 5 Gy (IR), immunostained with anti-g- H2AX. Bars: $10 \mu \mathrm{m}$. Representative immunoblot reporting the expression of $\mathrm{g}-\mathrm{H} 2 \mathrm{AX}$ in SW620 control cells, and cells 
irradiated with 5 Gy. C. Representative confocal microscopy images of SW620 control cells (CTR), and cells treated with hydroxyurea (HU) or doxorubicin (DOXO), immunostained with anti-g-H2AX. Bars: 10 $\mu \mathrm{m}$. Representative immunoblot of SW620 cells (CTRL), and cells treated with increasing concentrations of HU and DOXO. Right panel: Representative immunoblot of total lysate (TL), cytosolic (cyt) and nuclear (N) fractions obtained from SW620 treated or not with $4 \mathrm{mM} \mathrm{HU}$ or $2 \mu \mathrm{M}$ DOXO.

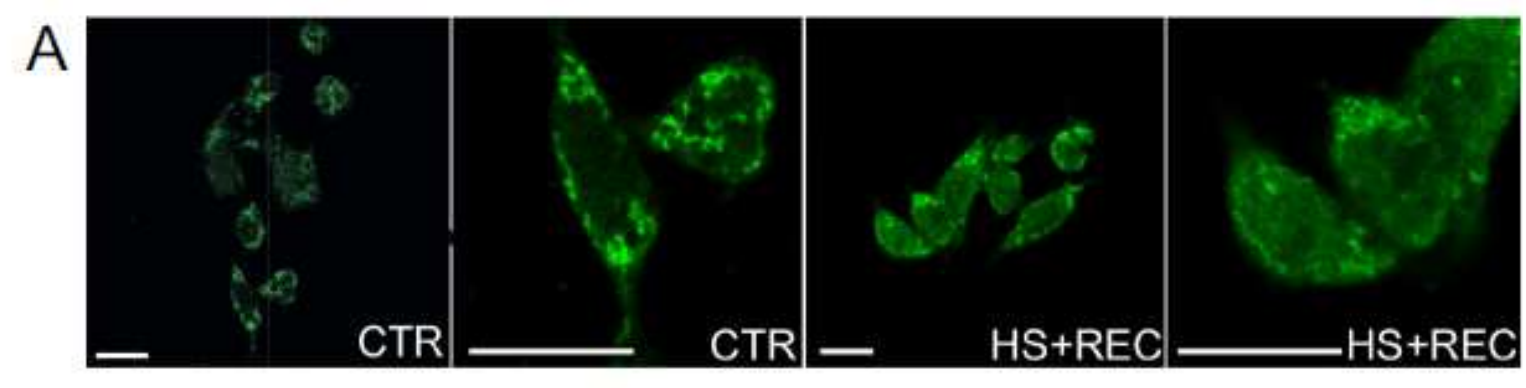

B

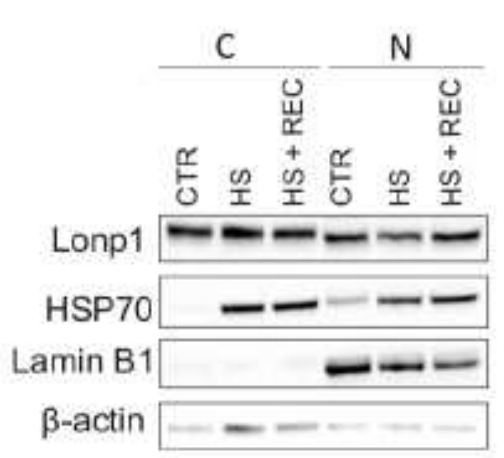

D

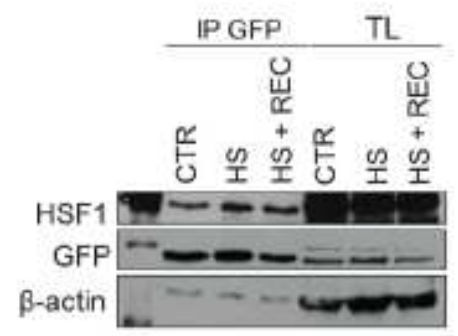

$\mathrm{F}$

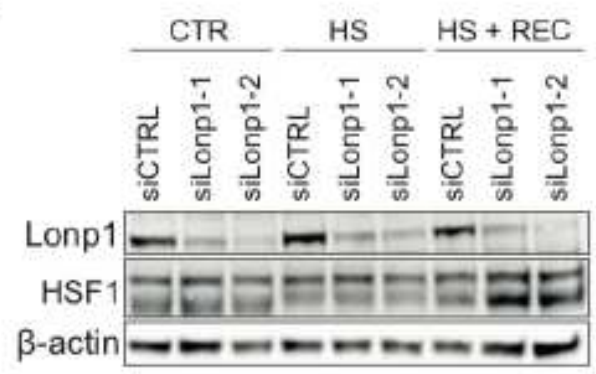

C

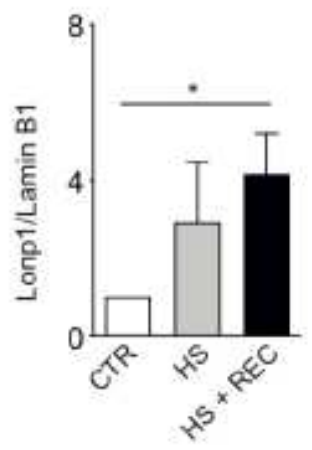

$E$

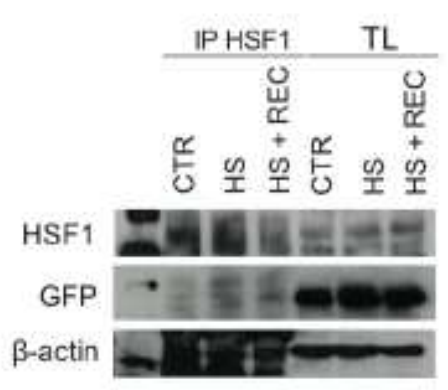

HSF1

GFP

$\beta$-actin

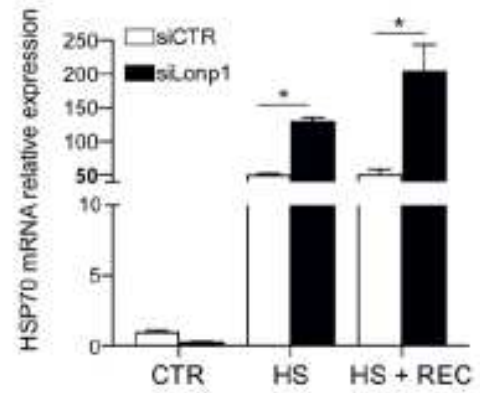


Lonp1 relocates in the nucleus in response to heat shock. A. Representative confocal microscopy images of SW620 cells after immunostaining with anti-Lonp1 antibody. Cells were heat-shocked (HS) at $42^{\circ} \mathrm{C}$ and then left to recover (HS+REC). B. Representative immunoblot showing Lonp1 expression in cytosolic and nuclear fractions from SW620 cells maintained at $37^{\circ} \mathrm{C}(\mathrm{CTR})$, at $42^{\circ} \mathrm{C}$ for $3 \mathrm{hrs}$ to induce heat shock (HS) and kept at $42^{\circ} \mathrm{C}$ for 3 hrs and then left for 1 hour at $37^{\circ} \mathrm{C}$ to recover (HS+REC). HSP70 was used as a positive control for HS treatment, and Lamin B1 and actin as markers for nuclear and cytosolic fractions, respectively. C. Histogram representing the relative levels of Lonp1 in nuclear fractions, obtained from three independent experiments. Data are shown as mean SD. $*=p<0.05$. D. Representative immunoprecipitation experiment showing the interaction between Lonp1-eGFP and HSF1 in SW620 cells after heat-shock (HS) and then left 3 hours at $37^{\circ} \mathrm{C}$ to recover (HS+REC). Lysates were immunoprecipitated with anti-eGFP and immunoblotted with anti-HSF1. E. Representative immunoprecipitation experiment showing the interaction between Lonp1-eGFP and HSF1 in SW620 cells after heat-shock (HS) and then left 3 hours at $37^{\circ} \mathrm{C}$ to recover (HS+REC). Lysates were immunoprecipitated with anti-HSF1 and immunoblotted with anti-eGFP. F. Representative immunoblot showing the quantification of HSF1 in SW620 cells transfected with scramble small-interfering RNAs (siCTRL) and cells transfected with small-interfering RNAs against Lonp1 (siLonp1 and siLonp2), after HS and HS+REC. G. Quantification of the mRNA levels of HSP70 in SW620 cells transfected with scramble small-interfering RNAs (SiCTRL) and cells transfected with small-interfering RNAs against Lonp1 (siLonp1), after HS and HS+REC. * $<<0.05$. 

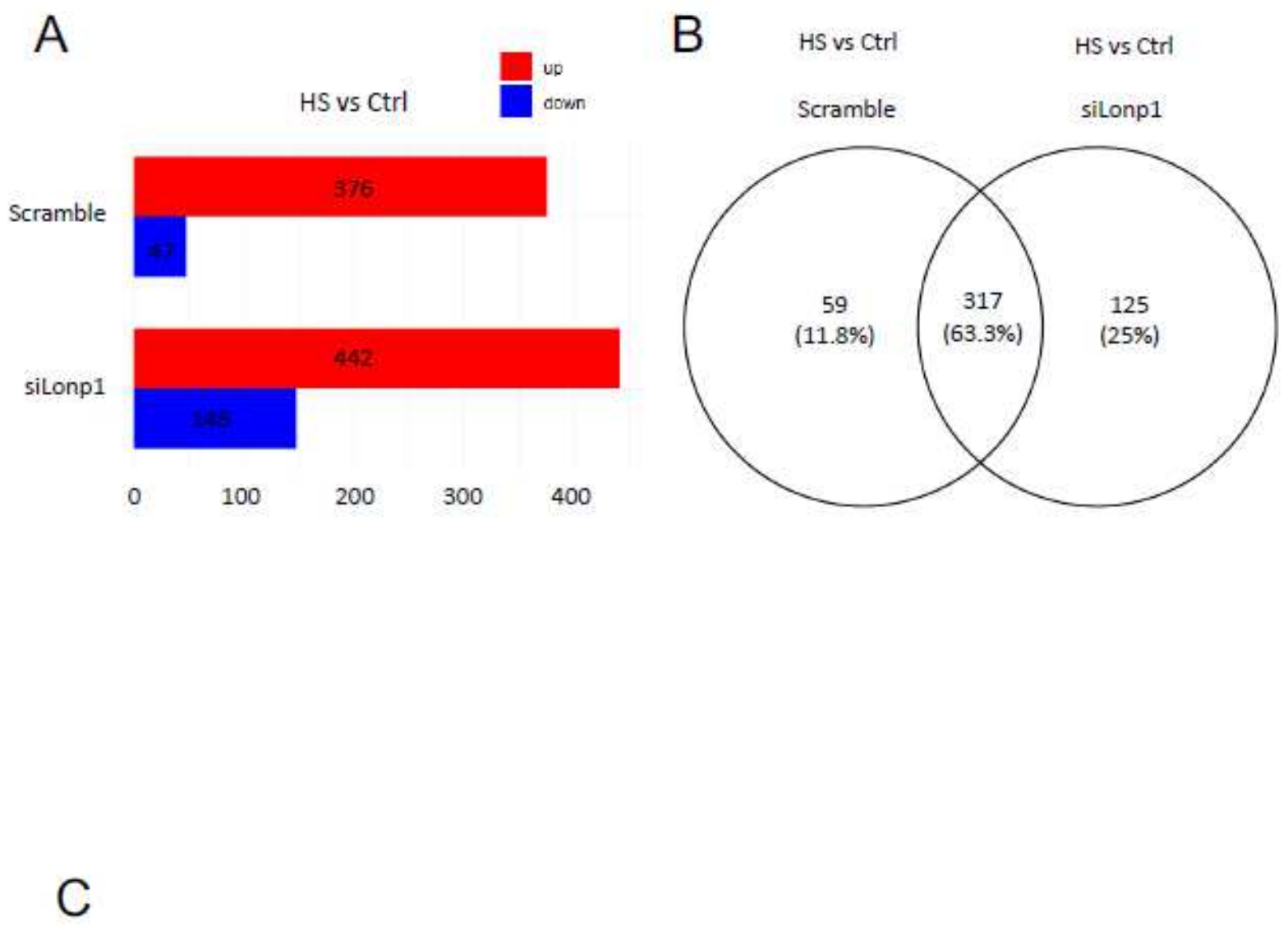

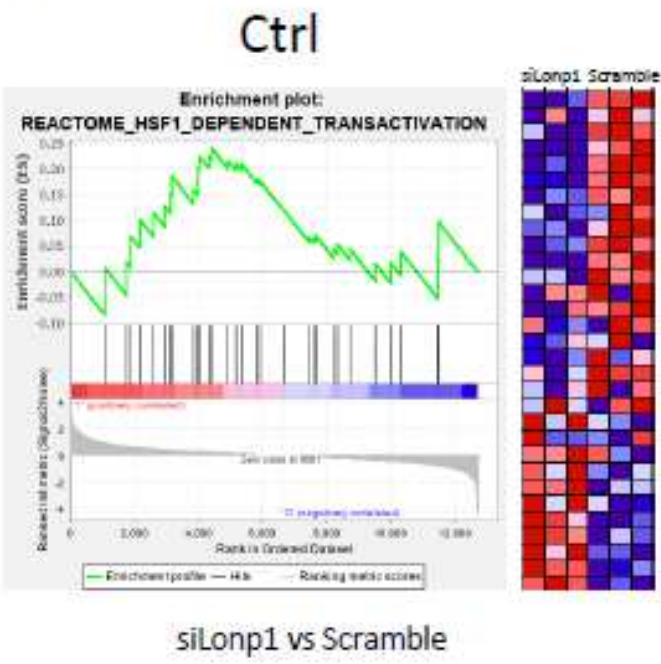

NES: $0.74 \quad$ FDR: 1

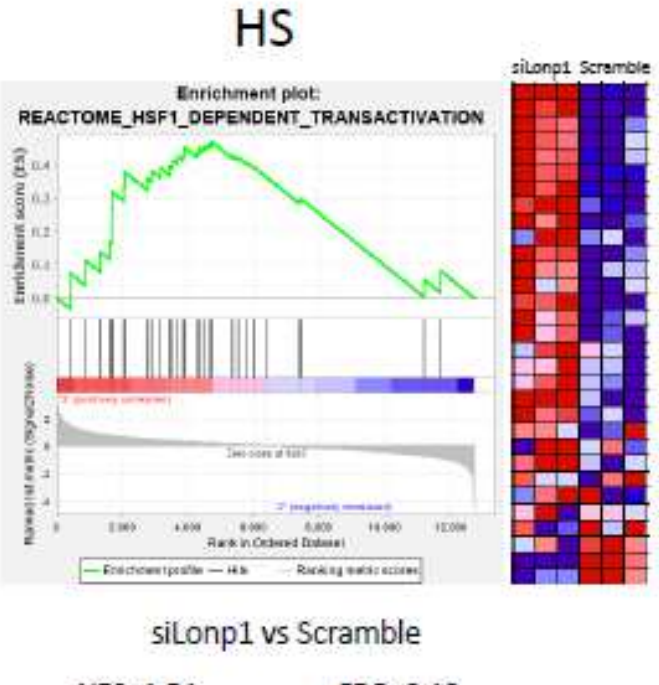

NES: 1.51

FDR: 0.19

\section{Figure 5}

Silencing of Lonp1 enhances HSF1-mediated response to HS. A. Number of genes significantly up- or down-regulated in SW620 cells transfected with small-interfering RNAs against Lonp1 (siLonp1) or with a scramble siRNA, kept at $37^{\circ} \mathrm{C}$ (Ctrl) or at $42^{\circ}$ for $1 \mathrm{~h}(\mathrm{HS})$. FDR q value $£ 0.05$ was used to identify differentially expressed genes, with fold change $(\mathrm{FC})>2$ for up-regulated and $\mathrm{FC}<2$ for down-regulated genes. B) Venn diagram showing the overlap between genes upregulated in HS, in cells where Lonp1 was 
silenced (siLonp1) or not (Scramble) in SW620 cells. C. Left panel: Gene set enrichment analysis (GSEA) plots for the "Reactome - HSF1 dependent transactivation" signature in SW620 cells transfected with small-interfering RNA against Lonp1 (siLonp1) in comparison with a scramble siRNA, kept at $37^{\circ} \mathrm{C}$. Right panel: Gene set enrichment analysis (GSEA) plots for the "Reactome - HSF1 dependent transactivation" signature in SW620 cells transfected with small-interfering RNA against Lonp1 (siLonp1) in comparison with a scramble siRNA, kept at $42^{\circ}$ for $1 \mathrm{~h}$. The Normalized enrichment score (NES) and FDR values for both analyses are reported.
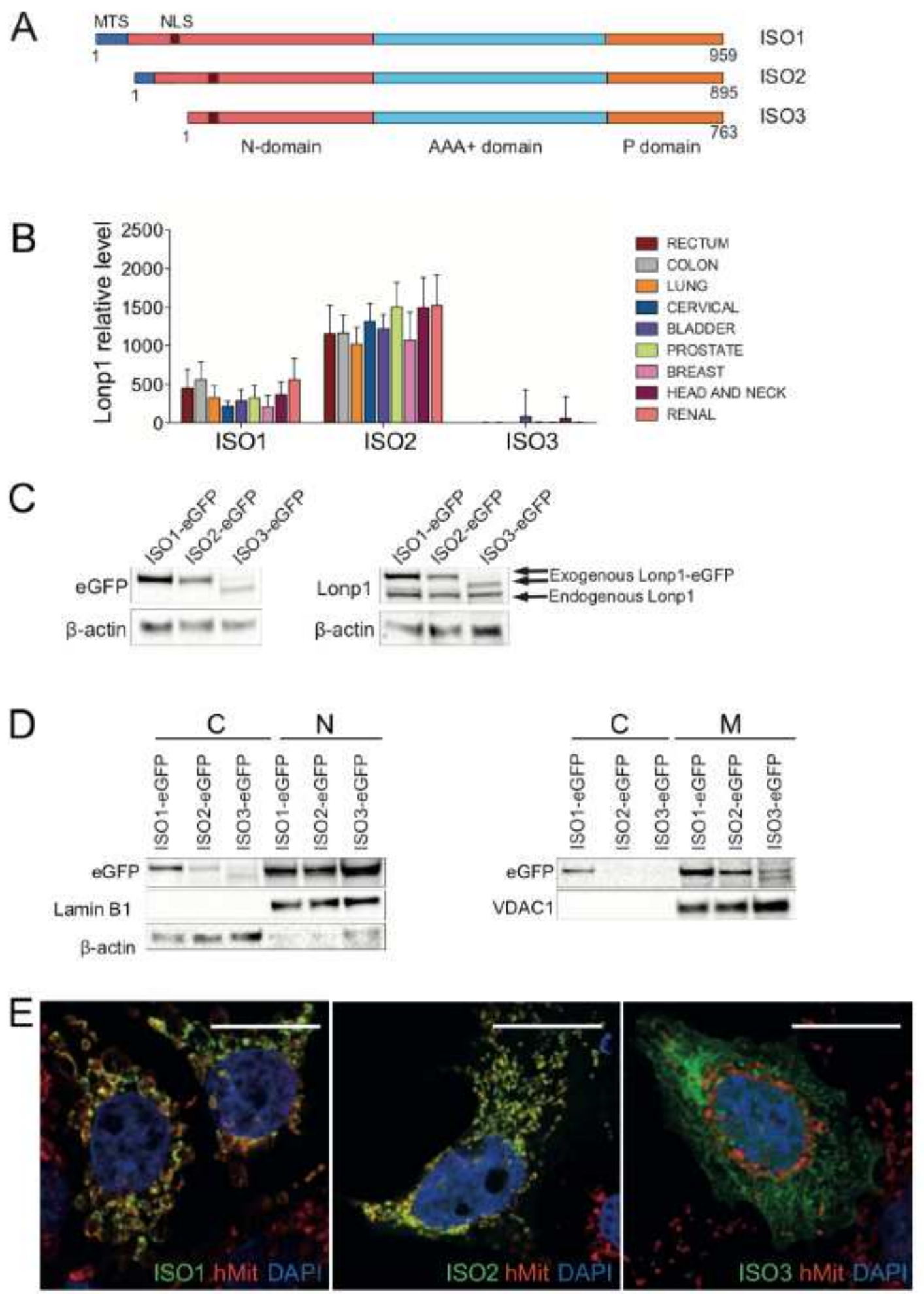


\section{Figure 6}

Human cells express three isoforms of Lonp1, whose subcellular localization is different. A. Diagram depicting the location of mitochondrial targeting sequence (MTS), of the nuclear localization signal (NLS), and of the N, AAA+ and P domains of three isoforms of human Lonp1. The length of the three isoforms is indicated. B. Histogram showing the relative expression levels of mRNAs encoding Lonp1 isoform-1 (ISO1), Lonp1 isoform-2 (ISO2), Lonp1 isoform-3 (ISO3) in normal tissue, obtained from TGCA Splicing Variant database (TSVdb). C. Representative immunoblot detecting Lonp1 using anti eGFP (left panel) and anti Lonp1 (right panel) Abs in cells transfected with Lonp1 ISO-eGFP, ISO2-eGFP or ISO3eGFP. The position of the bands corresponding to the exogenous, eGFP-tagged form and the endogenous form of Lonp1 is indicated. D. Representative immunoblots showing Lonp1 expression in cytosolic (C) and nuclear (N) fractions (left panel) and cytosolic (C) and mitochondrial (M) fractions (right panel) in SW620 cells transfected with constructs bearing Lonp1 isoforms tagged at C-term with enhanced green fluorescent protein (eGFP). Lonp1 has been detected using anti-eGFP antibody. E. Representative confocal microscopy images of HeLa cells transfected with three different constructs bearing Lonp1 isoforms tagged at C-term with enhanced green fluorescent protein (eGFP), namely Lonp1-ISO1, Lonp1ISO2 or Lonp1-ISO3. Mitochondria were stained with anti-hMit and nuclei were counterstained with DAPI. 
A

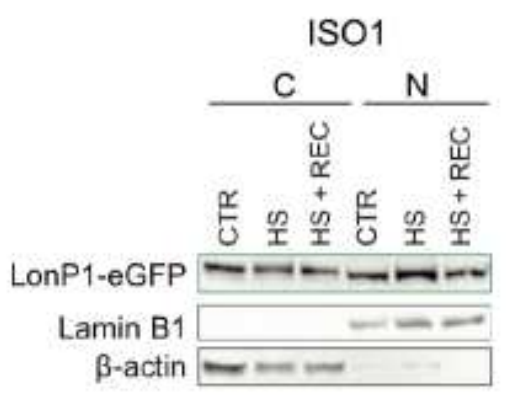

ISO2

ISO3
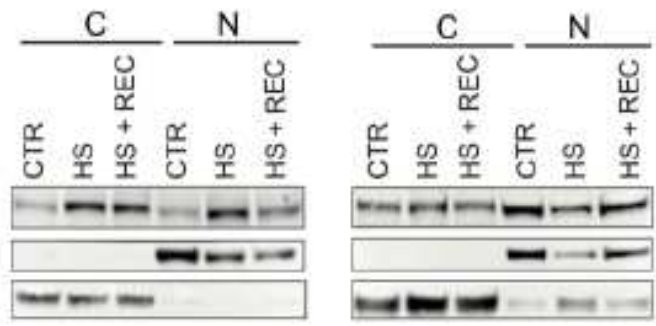

ISO1

B

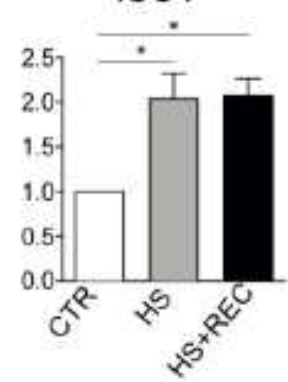

ISO2

ISO3

C
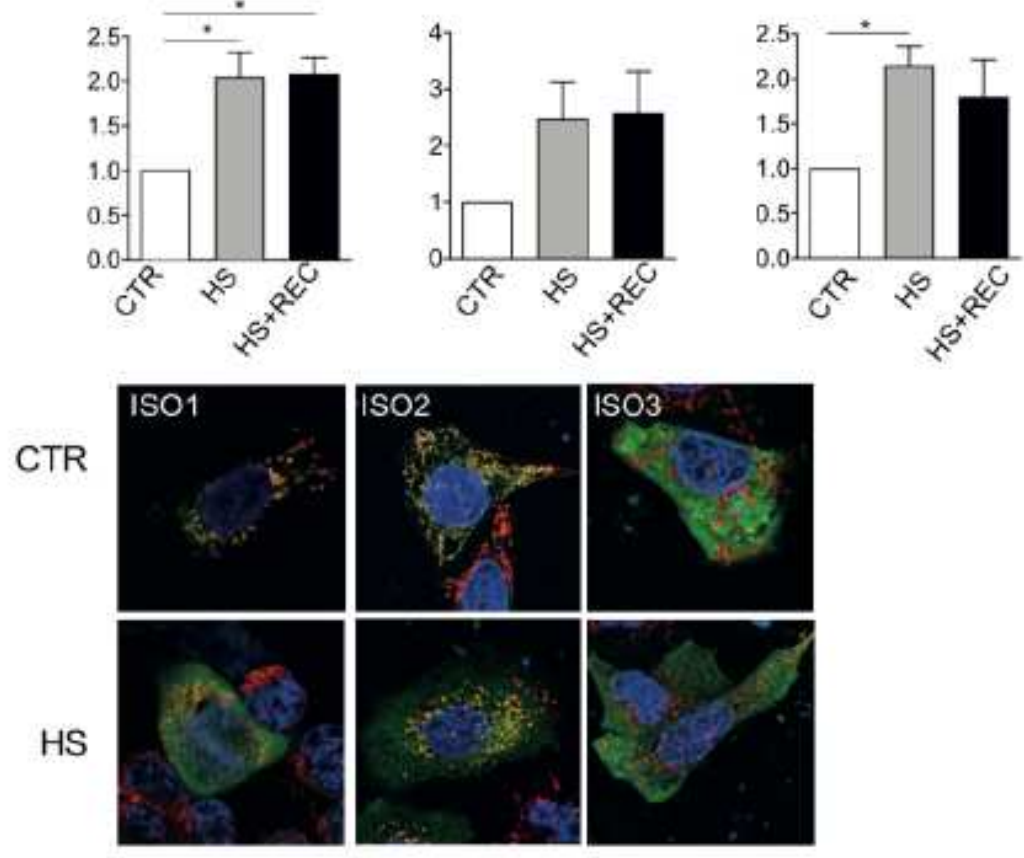

\section{D}

ISO1
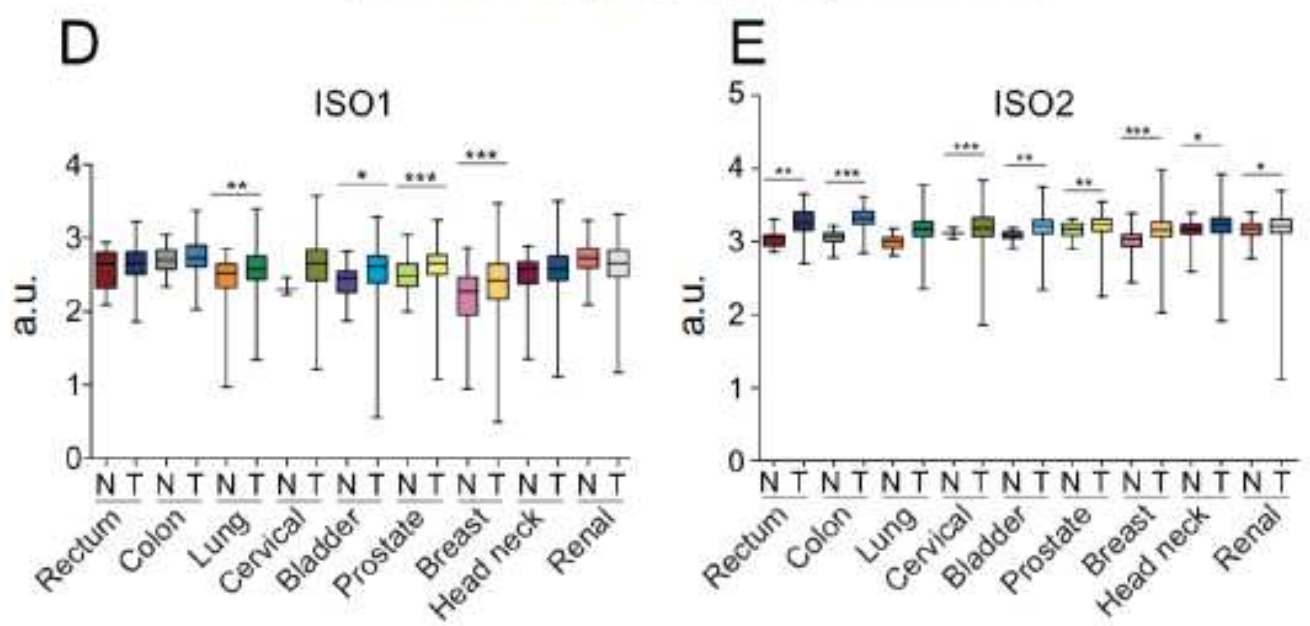

\section{Figure 7}

Lonp1 IS01 increases in the nucleus in response to heat shock A. Representative immunoblot detecting Lonp1 in cells transfected with Lonp1 ISO1-eGFP, ISO2 -eGFP or ISO3-eGFP in untreated conditions (CTR), undergoing HS for 3 hours (HS) and HS for 3 hours followed by recovery (HS+REC). B. Histograms showing the relative expression levels of Lonp1 ISO1, ISO2, and ISO3 in in untreated cells (CTR), cells undergoing HS for 3 hours (HS) and HS for 3 hours followed by recovery (HS+REC). Data are reported as 
mean $\pm S D$ of three independent experiments. C. Representative confocal microscopy images of SW620 cells transfected with Lonp1 ISO1-eGFP, ISO2 -eGFP or ISO3-eGFP, in untreated conditions (CTR), undergoing $\mathrm{HS}$ and $\mathrm{HS}$ followed by recovery (HS+REC). D. Histogram showing the relative expression levels of Lonp1 ISO1 mRNA in the indicated primary solid tumours $(\mathrm{T})$ and the normal (N) counterparts, as obtained from TGCA Splicing Variant database (TSVdb). Rectum: rectum adenocarcinoma; Colon: colon adenocarcinoma; Lung: lung adenocarcinoma; Cervical: cervical squamous cell carcinoma and endocervical carcinoma; Bladder: bladder urothelial carcinoma; Prostate: prostate adenocarcinoma; Breast: breast invasive carcinoma; Head and neck: head and neck squamous cell carcinoma; Renal: renal clear cell carcinoma. Data are in log scale; mean, 24 and 75 percentile, max and min values are shown. ${ }^{*}=p<0.05 ;{ }^{* *}=p<0.01 ;{ }^{* *}=p<0.0001$. E. Histogram showing the relative expression levels of Lonp1 ISO2 mRNA in the indicated primary solid tumours $(\mathrm{T})$ and the normal $(\mathrm{N})$ counterparts, as obtained from TGCA Splicing Variant database (TSVdb). Rectum: rectum adenocarcinoma; Colon: colon adenocarcinoma; Lung: lung adenocarcinoma; Cervical: cervical squamous cell carcinoma and endocervical carcinoma; Bladder: bladder urothelial carcinoma; Prostate: prostate adenocarcinoma; Breast: breast invasive carcinoma; Head and neck: head and neck squamous cell carcinoma; Renal: renal clear cell carcinoma. Data are in log scale; mean, 24 and 75 percentile, max and min values are shown. $*=p<0.05 ; * *=p<0.01 ; * \star *=p<0.0001$.

\section{Supplementary Files}

This is a list of supplementary files associated with this preprint. Click to download.

- SUPPLEMENTARYFIGURESCMLS.pdf

- Supplementarytable1.docx

- Supplementarytable2.docx

- Supplementarytable3.docx

- Supplementarytable4.docx 